\title{
Genomic structure and expression of Jmjd6 and evolutionary analysis in the context of related JmjC domain containing proteins Phillip Hahn ${ }^{\dagger 1}$, Jens Böse ${ }^{\dagger 1}$, Stefanie Edler $^{1}$ and Andreas Lengeling*1,2
}

Address: ${ }^{1}$ Research Group Infection Genetics, Department of Experimental Mouse Genetics, Helmholtz Centre for Infection Research, D-31824 Braunschweig, Germany and ${ }^{2}$ The Roslin Institute and Royal (Dick) School of Veterinary Studies, Easter Bush Veterinary Research Centre, The University of Edinburgh, Roslin, EH25 9RG, UK

Email: Phillip Hahn - Phillip.Hahn@helmholtz-hzi.de; Jens Böse - Jens.Boese@leica-microsystems.com; Stefanie Edler - Stefanie.Edler@helmholtz-hzi.de; Andreas Lengeling* - Andreas.Lengeling@ed.ac.uk

* Corresponding author †Equal contributors

Published: 18 June 2008

BMC Genomics 2008, 9:293

doi:10.|I|86/|47|-2|64-9-293

Received: 22 June 2007

Accepted: 18 June 2008

This article is available from: http://www.biomedcentral.com//47/-2/64/9/293

(c) $2008 \mathrm{Hahn}$ et al; licensee BioMed Central Ltd.

This is an Open Access article distributed under the terms of the Creative Commons Attribution License (http://creativecommons.org/licenses/by/2.0), which permits unrestricted use, distribution, and reproduction in any medium, provided the original work is properly cited.

\begin{abstract}
Background: The jumonji $C(\mathrm{Jmj} C)$ domain containing gene 6 (Jmjd6, previously known as phosphatidylserine receptor) has misleadingly been annotated to encode a transmembrane receptor for the engulfment of apoptotic cells. Given the importance of JmjC domain containing proteins in controlling a wide range of diverse biological functions, we undertook a comparative genomic analysis to gain further insights in Jmjd6 gene organisation, evolution, and protein function.

Results: We describe here a semiautomated computational pipeline to identify and annotate JmjC domain containing proteins. Using a sequence segment $\mathrm{N}$-terminal of the Jmjd6 JmjC domain as query for a reciprocal BLAST search, we identified homologous sequences in 62 species across all major phyla. Retrieved Jmjd6 sequences were used to phylogenetically analyse corresponding loci and their genomic neighbourhood. This analysis let to the identification and characterisation of a bi-directional transcriptional unit compromising the Jmjd6 and I/l0005A03Rik genes and to the recognition of a new, before overseen Jmjd6 exon in mammals. Using expression studies, two novel Jmjd6 splice variants were identified and validated in vivo. Analysis of the Jmjd6 neighbouring gene I I / 0005A03Rik revealed an incident deletion of this gene in two out of three earlier reported Jmjd6 knockout mice, which might affect previously described conflicting phenotypes. To determine potentially important residues for Jmjd6 function a structural model of the Jmjd6 protein was calculated based on sequence conservation. This approach identified a conserved double-stranded $\beta$-helix (DSBH) fold and a $\mathrm{HxDx}_{\mathrm{n}} \mathrm{H}$ facial triad as structural motifs. Moreover, our systematic annotation in nine species identified $3 / 3 \mathrm{DSBH}$ fold-containing proteins that split into 25 highly conserved subgroups.
\end{abstract}

Conclusion: We give further evidence that Jmjd6 most likely has a function as a nonheme-Fe(II)2-oxoglutarate-dependent dioxygenase as previously suggested. Further, we provide novel insights into the evolution of Jmjd6 and other related members of the superfamily of JmjC domain containing proteins. Finally, we discuss possibilities of the involvement of Jmjd6 and / / I0005A03Rik in an antagonistic biochemical pathway. 


\section{Background}

The jumonji C (JmjC)-domain containing proteins are an extending family of redox enzymes that catalyse a wide range of oxidation reactions. These proteins are found in all living organisms and are characterised by sharing the highly conserved JmjC domain. This domain was first described by Takeuchi and colleagues, who isolated a gene in the mouse which they called jumonji (meaning cruciform in Japanese), in regard to an abnormal phenotype visible in mutant embryos during neural plate development [1]. Since then, the JmjC domain has found its entry into public domain databases and many other proteins containing this domain have been annotated or identified subsequently [2-5]. Currently, more then 11,000 sequence entries of JmjC domain containing proteins are present in Uniprot, PFAM, Interpro and SMART, thus demonstrating the tremendous expansion of members in this protein superfamily. Structural studies showed that the JmjC domain has a very characteristic topology. It forms a double-stranded $\beta$-helix (DSBH) fold, also known as jelly-roll motif, that normally consists of eight antiparallel $\beta$-strands [6]. This DSBH topology is characteristic for the cupin metalloenzymes [7] and for the JmjC domain containing proteins [3]. Cupin metalloenzymes constitute a large and diverse superfamily of proteins with enzymatically active and non-active members. They contain at least one DSBH or jelly-roll motive. Secondary structure elements that surround the DSBH are used further to define different subfamilies (for review see [6]). The DSBH fold in JmjC domain containing proteins can form an enzymatically active pocket by coordinating di-iron (Fe(II)) and the co-substrate 2-oxoglutarate (2OG). In almost all cases, the two-electron oxidation of the "prime" substrate is coupled to the conversion of 2OG into succinate and $\mathrm{CO}_{2}$ [8]. Therefore, this class of enzymes is also known as nonheme-Fe(II)-2-oxoglutarate-dependent dioxygenases.

Oxidative reactions catalysed by 2OG-dependent dioxygenase are critical steps in the biosynthesis of metabolites, or post translational modification of interacting target proteins involving hydroxylation or demethylation and DNA/RNA repair of N-methylated nucleic acids [6,9]. As such, 2OG-dependent dioxygenases are of immanent biological importance. A number of JmjC domain containing proteins are involved in regulating key developmental processes during mammalian embryogenesis by acting as transcription factors. An example of this subgroup of JmjC domain containing proteins is jumonji itself (now called Jarid2), which can act as a transcriptional repressor. Jarid2 inhibits cyclin D1 transcription during heart and brain development and thereby controls cellular proliferation and differentiation in these organs during morphogenesis [10-12]. Other examples of JmjC-domain proteins that function as transcriptional repressors are the retinoblast- oma-binding protein 2 (Jarid1a, also known as RBP2), which is required for the differentiation of various cell types including muscle, bone and myeloid cells [13] and hairless, a nuclear co-repressor of multiple nuclear hormone receptors such as the vitamin $\mathrm{D}$ receptor, the thyroid hormone receptor and the retinoic acid-receptorrelated orphan receptor $\alpha$ [14-16]. Mutations in hairless result in humans and mice in alopecia, a form of hereditary hair loss due to defects in the regeneration of hair follicle cells [17-19]. More recently, JmjC-domain containing proteins have gained emerging importance in histone modification and chromatin regulation. The JmjC domain containing histone demethylases (JHDMs) can catalyse lysine demethylation of histones through an oxidative reaction that requires iron $\mathrm{Fe}$ (II) and 2OG. Histone methylation marks have important roles in regulating gene expression and are central to the control of epigenetic information that regulates cell fate and identity [20]. Until now, JHDMs have been reported to be capable to reverse H3K36 (JHDM1/FBXL11, [21], JMJD2A, [22,23], Rph1, [24,25], Jhd2, [25], Gis1, [25], Jhd1, [25]), H3K9 (JHDM2A/JMJD1A, [26]), and both H3K9 and H3K36 methylation (JHDM3A to JHDM3D/JMJD2A to JMJD2D, [23,27-30]). In addition, the JARID subfamily of JmjCdomain containing proteins have been shown to possess H3K4 demethylase activity in different species (JARID1A/ RBP2, [31,32], JARID1C/SMCX, [31,33], JARID1D/ SMCY, [34], JARID1B/PLU-1/Yjr119Cp/Jhdp2, [31,35,36], Lid, [26,37-39], and JMJ2 [40]). Most recently, the JmjC-domain containing proteins UTX and JMJD3 have been shown to function as H3K27 specific demethylases and demonstrated to play an important role in animal body patterning and regulation of inflammatory responses [41-44]. Through this activity the JHDMs have been postulated to regulate diverse cellular processes including control of cell proliferation, heterochromatin assembly and gene expression [26,28,30,34,45,46]. However, for most of the newly discovered JHDMs gene ablation studies in vivo have as yet not been carried out leaving the possibility open that in addition to histones other substrates might be targets of these proteins.

Many of the different classes of JmjC domain containing proteins possess complex domain architectures by having at least one additional protein domain besides the JmjC domain. Here, the JHDMs and Jarid proteins are good examples. In addition to the JmjC domain they have PHD, Tudor, Bright/Arid and zinc finger domains, which have recently been shown to interact with methylated histone tails and are required for their catalytic function $[33,38,47]$. In contrast, other members in this protein superfamily have no other recognisable protein domain apart from the JmjC domain. Important members of this subgroup of JmjC domain only proteins are the 2OGdependent dioxygenase factor inhibiting HIF (Hif1an, 
also known as FIH) and the PHD hydroxylases, which are involved in the maintenance of oxygen homeostasis. Hiflan was the first structurally resolved mammalian JmjC domain containing protein $[48,49]$ and was shown to function as an asparagine hydroxylase for the hypoxia inducible factor $\alpha(\mathrm{HIF} 1 \alpha)$, a transcription factor that is central to oxygen homeostasis in both physiological and pathophysiological processes (for review see [50]). The PHD hydroxylases PHD1 to PHD3 also target HIF1 $\alpha$ by hydroxylating proline residues. Under normal oxygen conditions the hydroxylation reactions carried out by PHDs induce the proteasomal degradation of the HIF1 $\alpha$ subunit, whereas under hypoxia HIF1 $\alpha$ is not hydroxylated and translocates from the cytoplasm to the nucleus where it activates transcription of genes needed for adaptation to low oxygen levels. AlkB is another member of the JmjC domain only proteins. As a 2OG-dependent oxygenase AlkB can repair DNA that is methylated at 1-methyladenine and 3-methylcytosine by oxidation of the methyl group [51,52]. AlkB and related proteins define a subfamily of small 2OG-dependent oxygenases that are involved in DNA and RNA repair [53].

In the past, we have analysed the function of the phosphati$d y l$ serine receptor (Ptdsr or also known as PSR or PtdSerR), a gene that also encodes a JmjC domain only protein $[54,55]$. First described as a cell surface receptor for the recognition and engulfment of apoptotic cells [56], recent studies have raised serious doubts about its involvement in apoptotic cell clearance. Five groups independently reported a nuclear localisation of Ptdsr, which clearly contradicts the hypothesis that the protein might function as a transmembrane receptor [57-61]. Furthermore, a comprehensive analysis carried out by us in Ptdsr-knockout mice demonstrated no impairment in the removal of apoptotic cells in Ptdsr-deficient embryos and macrophages ([54] for Ptds ${ }^{t m 1 G b f}$ ) thereby contradicting two previous studies that reported defects in apoptotic cell clearance in two other Ptdsr-knockout mice ([62] for Ptdsrtm1Flv, and [63] for Ptdsrtm1Ysfk). All studies involving $P t d s r$-knockout mice conclusively demonstrated that the gene is essential for normal development. Ptdsr-deficient mice are dying at birth and display severe differentiation defects in many tissues such as brain, eyes, lung, kidney, liver and intestine at different stages of embryogenesis $[54,62,63]$. Furthermore, Ptdsr plays an important role during heart development and ablation of Ptdsr function is associated with complex cardio-pulmonary malformations that resemble the human congenital heart syndrome Tetralogy of Fallot [55].

Although Ptdsr is clearly an important differentiation-promoting gene during embryogenesis its molecular function is still unknown. Based on a bioinformatic analysis of the cloned Hydra homologue PSR, Cikala and colleagues were the first to suggest that the protein might function as a 2OG-dependent dioxygenase that is capable of modifying unknown nuclear targets [57]. In recognition of the still unknown function of the "phosphatidylserine receptor gene" the International Committee for Standardized Genetic Nomenclature in Mice (ICSGNM) has most recently revised its name. Ptdsr has been renamed into jumonji domain containing gene 6 (Jmjd6). We will obey the guidelines of the ICSGNM and use this name and the gene symbol Jmjd6 throughout the remainder of this article.

To gain more insights into possible Jmjd 6 functions using sequence homology approaches, we developed a semiautomated process to extract all available Jmjd6 homologous protein and nucleic acid sequences from public databases. This comprehensive data set was used to identify proteins with sequence homology to Jmjd6 in 62 species across all major living phyla. In addition, this allowed us to identify new Jmjd6/JMJD6 splice variants in mice and humans and to analyse the genomic neighbourhood of the gene in vertebrates. Immediately downstream of Jmjd6 we found the gene 1110005A03Rik, which is predicted to encode a putative methyltransferase, which is as yet not further biochemically characterised. Interestingly, we found 1110005A03Rik to be affected by deletions in two previously reported Jmjd6 knockout mice. To predict possible structure-function relationships of Jmjd6, we employed a comparative modelling approach of the three-dimensional structure of the protein and annotated probable functional residues based on sequence conservation. To integrate Jmjd6 into the superfamily of JmjCdomain containing proteins, we assembled a non-redundant set of 313 DSBH-domain containing proteins by using our semiautomatic retrieval script and grouped these according to phylogenetic relationships. Taken together, our results further support a conserved enzymatic function of Jmjd6 most likely as a classical nonheme, $\mathrm{Fe}(\mathrm{II})-$ and 2OG-dependent dioxygenase. It also shows that Jmjd6 is closely related to Jmjd4 and JFP6, and constitutes together with these two proteins a subgroup of JmjC domain containing proteins. Moreover, we identified in protista, fungi and plants proteins with sequence homology to Jmjd6 that diverge from the classical "JmjConly domain" structure. Some of these proteins have in comparison to the animalia Jmjd6 extended C-terminal regions that contain additional domains that might have been lost during evolution.

With these approaches we establish here an overall framework of information that will facilitate not only further research regarding the functional characterisation of Jmjd6 but also of other members within the DSBHdomain containing superfamily of proteins. 


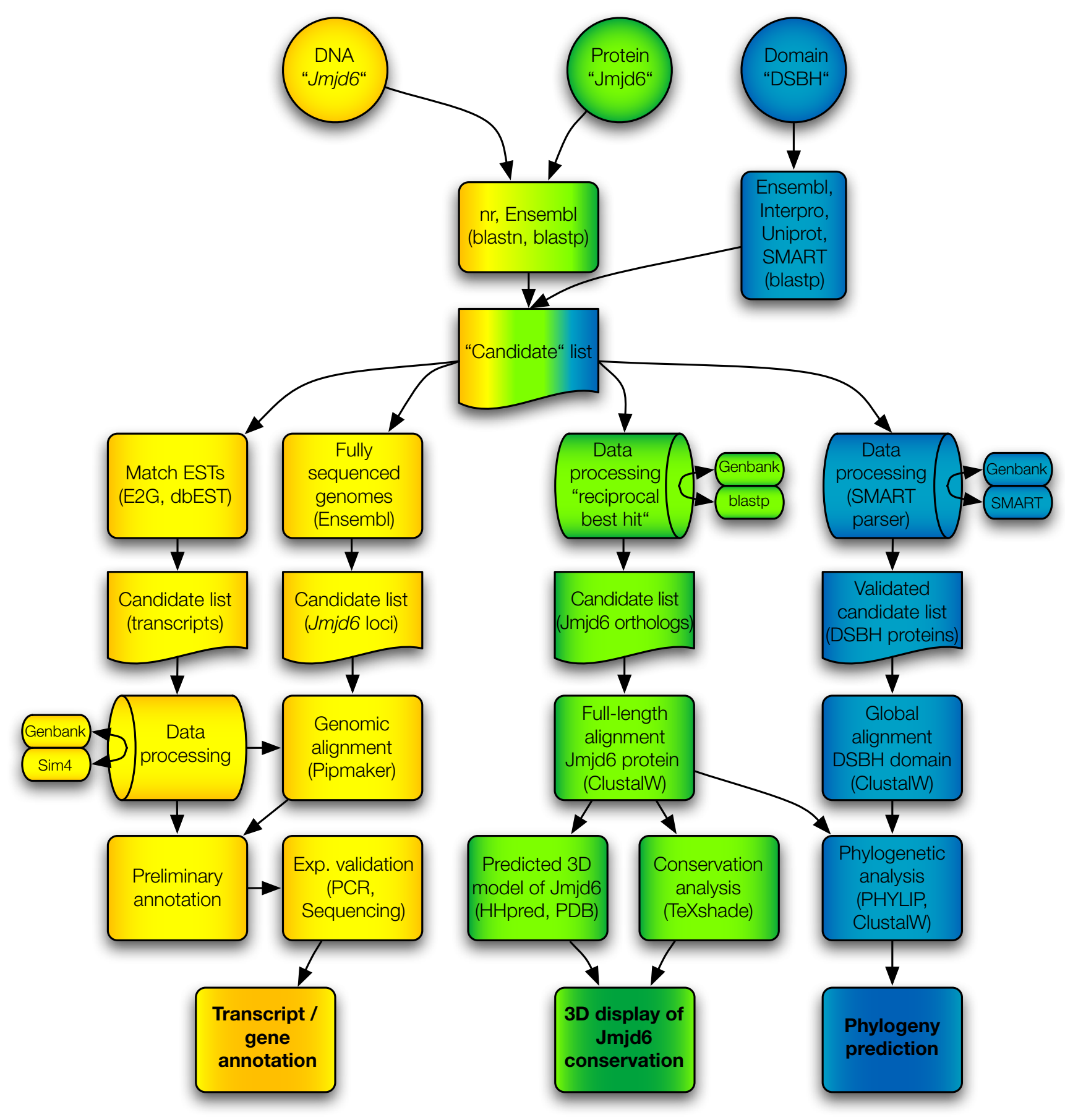

Figure I

Computational pipeline flow used for the retrieval and analysis of DSBH/JmjC-domain containing proteins. It contains three parallel procedures. Indicated in yellow colour are searches and analysis approaches based on DNA sequences, data processing approaches based on protein sequences are coloured green, and phylogenomic analysis methods based on DSBH protein domains are shown in blue. Initial queries are depicted as circles, databases as ovals, candidate gene and protein lists as cut-off boxes, and methods/analysis tools as normal boxes, respectively. Barrels indicate Perl script based analysis of retrieved results. Candidate lists of the individual workflows are shown and the final results of the analysis are depicted in bold. Arrows indicate the flow of information and the connections between different search routines and methods. Sources of queried databases and the analysis software used are given in the respective box elements. 


\section{Results \\ Retrieval and analysis of a comprehensive set of Jmjd6 orthologs and other JmjC domain containing proteins}

We developed a semiautomatic process to extract all available Jmjd6 homologous protein and nucleic acid sequences from public databases. The computational pipeline consists of three parallel procedures based on "DNA", "protein", and "protein domain" searches (Figure $1)$. With this approach, we generated a candidate list of 11.376 sequence entries including all putative Jmjd6 orthologs and all JmjC/DSBH domain containing proteins present in Genbank, Ensembl, Uniprot, PFAM, Interpro, and SMART, independent of their annotation. The goal of our semiautomated computational pipeline was then to identify and systematically analyse all orthologous Jmjd6 loci, all transcripts encoded by the murine and human Jmjd6/JMJD6 loci, the degree and distribution of sequence conservations in Jmjd6 proteins, and their phylogenetic relationship to other JmjC/DSBH domain containing proteins. In the end, the combination of these analysis approaches enabled us to identify 62 proteins with significant sequence homology to Jmjd6 and to establish a set of 313 non-redundant DSBH domain containing proteins in human and eight model organisms.

We started the "DNA" sequence based retrieval and analysis approach (depicted in yellow in Figure 1) by identification of all mouse Jmjd6 homologous sequences available in Ensembl (release 44). This candidate loci list contained homologous Jmjd6 loci in fully annotated genomes as well as Jmjd6 sequence fragments from ongoing sequencing projects. To identify conserved coding and non-coding sequences within these genomic regions we undertook a PipMaker analysis of retrieved sequences that showed a similar genomic organisation in comparison to the murine Jmjd6 locus. Next, we mapped all available murine and human ESTs in dbEST to the murine and human Jmjd6/JMJD6 loci, respectively, using the program e2g. Extensive validations of each EST employing Sim4 with our own perlscript for data retrieval, handling, examination and sorting of the results led to a comprehensive analysis of the murine and human Jmjd6/JMJD6 loci and a detailed annotation of all encoded transcripts.

To identify all Jmjd6 orthologs, we employed a blastp search against Ensembl and nr databases using a unique $\mathrm{N}$-terminal sequence of the murine Jmjd6 protein as a template ("protein" based search and analysis, depicted in Figure 1 in green). The candidate matches were individually verified in a Mus musculus restricted blastp database search using the reciprocal best BLAST hit criterion and a perlscript for automatic data processing. These led to the identification of Jmjd6 proteins in other species that are closely related to and might be orthologs of the murine Jmjd6 protein. A full length ClustalW alignment of pro- teins with significant homology to Jmjd6 was processed using a TeXshade based conservation analysis and also as input for a 3D structure prediction of the catalytic core domain. By combining these results, we developed a novel way of predicting residues that are most likely essential for the catalytic activity of the protein based on their degree of conservation.

To analyse the phylogenetic evolution of the Jmjd6 JmjC domain, we developed SMART-parser. SMART and SMART-parser were used to analyse the domain composition and extract the sequence information from a set of sequences that was derived from the Ensembl, Interpro, Uniprot and SMART databases ("DSBH-domain" routine, depicted in Figure 1 in blue). This set comprises all JmjC, Pfam-JmjC, Blast-JmjC-, P4Hc-, Pfam-2OG-FeII-Oxyand Pfam-PhyH-domain containing sequences from human, mouse, zebrafish, pufferfish, nematode, fruit fly, and yeast. These different domain names have been used in the past to annotate the JmjC domain in DSBH fold containing proteins. The SMART-parser verified sequences were used as an input for a global ClustalW alignment. A PHYLIP processing of all ClustalW alignments allowed the analysis of the Jmjd6 phylogeny in relationship to all other identified DSBH domain containing proteins.

\section{Comparative analysis of Jmjd6 homologous loci in vertebrates}

In the mouse, Jmjd6 is localised in a gene dense region on distal chromosome 11 . Using different gene targeting strategies three loss-of-function alleles of Jmjd6 have been generated that display conflicting phenotypes [54,62,63]. To analyse the immediate genomic neighbourhood of Jmjd6 and to identify all transcripts encoded by the locus, we undertook a comparative sequence analysis of this gene region in different species. In Ensembl, the mouse Jmjd6 gene is annotated to consist of six exons compromising $6 \mathrm{~kb}$ (Ensembl release 44). Immediately downstream of Jmjd6 two genes are located, 1110005A03Rik, an expressed sequence tag (EST) encoding a putative arginine protein methyltransferase [64], and Sfrs2, encoding the splicing factor SC 35 [65]. To analyse the genomic region encompassing Jmjd6 and 1110005A03Rik in more detail, we performed a comprehensive database screen. First, we searched all fully sequenced genomes present in Ensembl build release 44 for an annotated Jmjd6 gene. We identified Jmjd6 homologous genes or genomic Jmjd6 fragments in 17 mammalian, 1 avian, 1 amphibian, 5 fish, 2 urochordata, and 4 invertebrate genomes (Table 1). The identified putative Jmjd6 orthologous loci were then searched for the presence of the 1110005A03Rik gene. In 22 out of the 30 analysed genomes we found both genes located close together. Remarkably, in all analysed invertebrate species Jmjd6 and 1110005A03Rik were not found to Colocalise despite the presence of both genes at other chro- 
Table I: Species distribution and chromosomal locations of Jmjd6 orthologs in mammalian and non-mammalian genomes

\begin{tabular}{|c|c|c|c|c|}
\hline Species name & Common name & Genomic location & Cluster with III $0005 A 03 R i k$ & Cluster with Sfrs2 \\
\hline Homo sapiens & Human & 17 & + & + \\
\hline Pan troglodytes & Chimp & 17 & + & + \\
\hline Macaca mulatta & Macaque & 16 & + & + \\
\hline Mus musculus & Mouse & 11 & + & + \\
\hline Rattus norvegicus & Rat & 10 & + & + \\
\hline Cavia porcellus & Guniea pig & Gene scaffold 591 & + & \\
\hline Spermophilus tridecemlineatus & Ground squirrel & Scaffold 5110 & + & \\
\hline Tupaia belangeri & Tree shrew & Scaffold 5346 & + & \\
\hline Canis familiaris & Dog & 18 & + & + \\
\hline Felis catus & Cat & Scaffold 295 & + & \\
\hline Bos taurus & Cow & 19 & + & + \\
\hline Myotis lucifugus & Microbat & Scaffold 462 & + & \\
\hline Erinaceus europaeus & Hedgehoc & Scaffold 670 & + & + \\
\hline Dasypus novemcinctus & Armadillo & Scaffold 584 & + & \\
\hline Echinops telfairi & Tenrec & Scaffold 620 & + & \\
\hline Monodelphis domestica & Opossum & 2 & + & + \\
\hline Ornithorhynchus anatinus & Platypus & Ultra 430 & + & \\
\hline Gallus gallus & Chicken & 18 & + & \\
\hline Xenopus tropicalis & Western clawed frog & Scaffold 178 & + & + \\
\hline Gasterosteus aculeatus & Stickleback & Group XI & + & \\
\hline Oryzias latipes & Medaka & Scaffold 1014 & & \\
\hline Tetraodon nigroviridis & Tetraodon & SCAF I 3844 & + & \\
\hline Takifugu rubripes & Fugu & Scaffold 519 & + & \\
\hline Danio rerio & Zebrafish & 3 & + & + \\
\hline Ciona intestinalis & Sea squirt & $3 p$ & & \\
\hline Ciona savignyi & Sea squirt & Reftig I & & \\
\hline Drosophila melanogaster & Fruitfly & $3 R$ & & \\
\hline Aedes aegypti & Aedes & Super contig 1.887 & & \\
\hline Anopheles gambiae & Mosquito & $\mathrm{x}$ & & \\
\hline Caenorhabditis elegans & C. elegans & IV & & \\
\hline
\end{tabular}

Chromosomal locations have been taken from ENSEMBL, + indicates the presences of $1 / 10005$ A03Rik or Sfrs2 in the immediate genomic neighbourhood of Jmjd6.

mosomal locations in the examined genomes. Next, we extracted 50 to $100 \mathrm{~kb}$ of genomic sequence encompassing the Jmjd6 locus and adjacent loci of these 22 species and used these for an initial PipMaker sequence analysis (data not shown). To carry this out, the mouse sequence was first analysed for repetitive sequence elements using RepeatMasker and secondly, the positions of all coding sequences/exons in Jmjd6, 1110005A03Rik, and Sfrs2 were identified and annotated using the BLAST algorithm. The masked and annotated mouse sequence was then finally used for interspecies pairwise alignment with the human, bovine, armadillo, opossum, zebrafish, chicken, western clawed frog, and puffer fish sequences using the program PipMaker [66]. These species were selected for the analysis either based on their large phylogenetic distance to the mouse or on the availability of high quality, full-coverage sequence information in the extracted regions. The resulting alignments of similar regions in the genomic sequences were summarised as "percent identity plot" (pip) displaying sequence identity in combination with the annotated repeats and transcriptional units (Figure 2). The pip shows a high degree of sequence conservation in the coding regions of the three genes and confirms the accuracy of the initial exon assignments annotated by Ensembl. However, a detailed inspection of the Jmjd6 locus identified further high-level conserved sequences (= $90 \%$ ) in between the annotated exons 4 and 5 (red overlay in Figure 2). This sequence conservation suggested the presence of an up to now overseen additional exon, at least in the mammalian species. In addition, the pip confirms the close association of the Jmjd6 and 1110005A03Rik genes in all analysed organisms. Jmjd6 and 1110005A03Rik are arranged in a head-to-head transcriptional orientation with a short intergenic distance of 65 bp between both genes. This suggests that these two genes build a conserved transcriptional unit that possibly shares a bi-directional promoter region. This is further supported by the presence of a conserved short non-coding sequence in between the translational start sites of these two genes (orange overlay in Figure 2). Interestingly, we observed not only a conserved association of the Jmjd6 and 1110005 A03Rik genes in this genomic region, but also of $S f r s 2$, which is present in this region in all species with the exception of the puffer fish (Figure 2). In the 


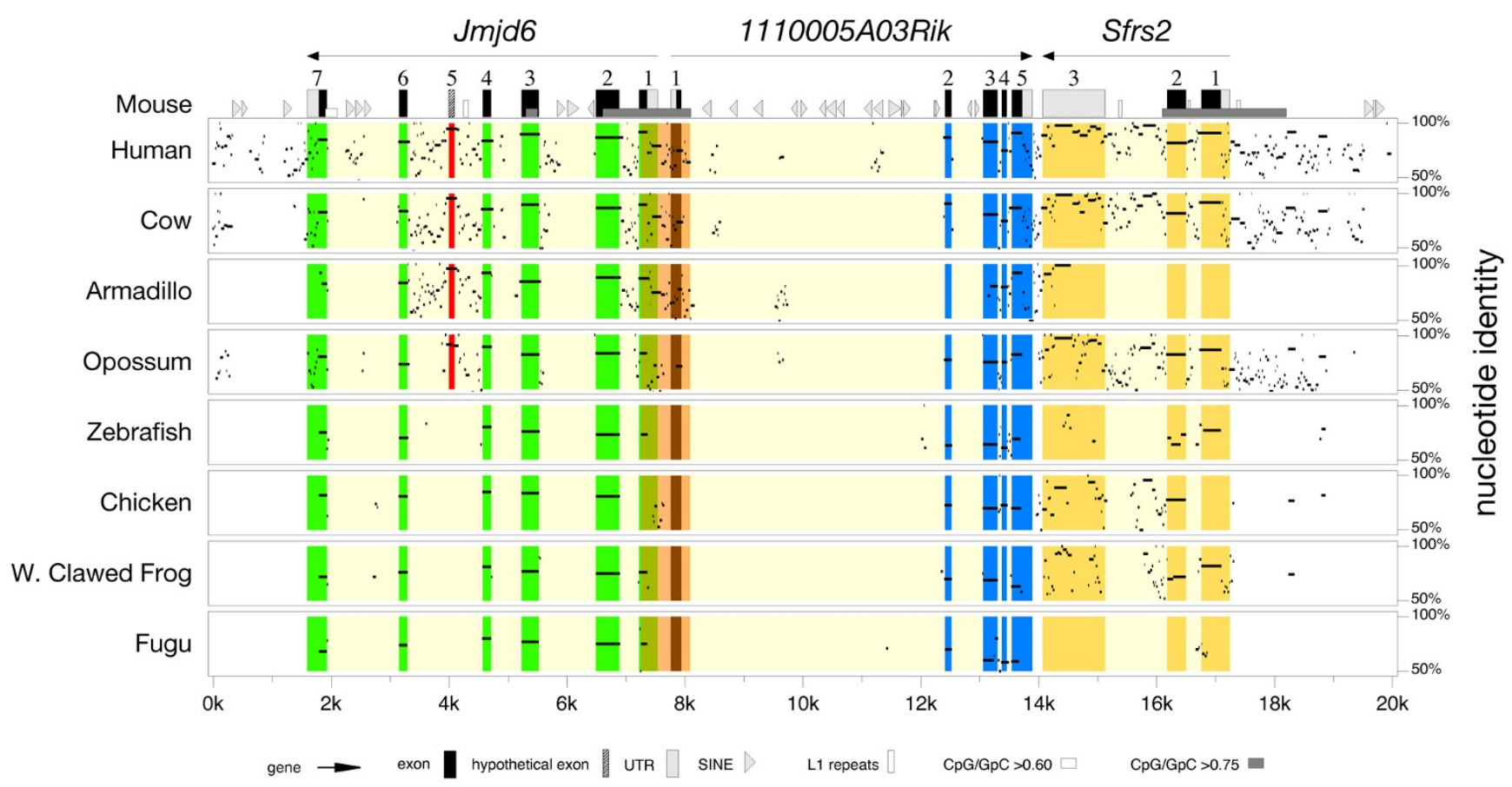

Figure 2

Percent identity plot of the chromosomal regions encompassing the Jmjd6 locus in vertebrate species. The genomic regions of eight vertebrate species identified as putative Jmjd6 orthologous loci were aligned with the mouse Jmjd6 locus and its neighbouring genes using PipMaker. Green, blue and yellow overlays highlight the conservation of the annotated exons/coding sequences of the individual genes found in the reference sequence. The orange overlay indicates conserved sequences of the first exons of the Jmjd6 and I / / 0005A03Rik genes and sequences outside of the coding elements. The red overlay marks a high-scoring segment in all analysed mammalian species supporting the presence of at least one additional exon (No. 5) that is not included in the current Ensembl gene annotation (build 44). Transcriptional orientations of the genes, their exons, UTRs, repetitive elements, and CPG islands are in the symbol key at the figure bottom.

mouse, Sfrs 2 is located $6.3 \mathrm{~kb}$ distal of Jmjd6 and $170 \mathrm{bp}$ distal to 1110005A03Rik (Figure 2).

To further test the hypothesis that an additional exon in Jmjd6 might generate alternative transcripts of this gene, we investigated all available mouse Jmjd6 EST data currently present in dbEST. Using e2g, a web-based server that allows an efficient mapping of large EST and cDNA datasets to genomic regions [67], all Jmjd6 ESTs were mapped to the mouse locus and adjacent regions. The resulting EST hits represent the entire putative Jmjd6 coding region including the transcribed and untranslated regions (UTRs). These cDNA sequences were then extracted and individually aligned with the mouse Jmjd6 genomic sequence using $\operatorname{Sim} 4$, a computer program that allows an efficient assignment of cDNAs to large genomic regions by accounting for the position of introns [68]. The Sim 4 validated EST sequences were then manually curated and all predicted Jmjd6 transcripts were assembled and annotated to the genomic locus. As a result of this analysis, we identified a new variant of exon 4 in $J m j d 6$ and found an additional, before overseen exon in the con- served region between exons 4 and 5. This allowed us, solely based on the EST data, to predict the presence of two novel Jmjd6 splice variants in addition to the main transcript of $1.4 \mathrm{~kb}$ currently annotated in Ensembl (Figure $3 \mathrm{~A}$ ). To validate in vivo the existence of the predicted splice variants, we performed RT-PCR expression analysis. Various major organs from adult mice as well as whole embryos at different developmental stages (E) were used for the analysis. RT-PCR amplification yielded three DNA fragments indicative for the predicted transcripts in all analysed samples at varying levels (Figure 3B). Taken together, this confirmed the computational prediction of two new Jmjd6 splice variants encoded by the mouse locus and demonstrated that $J m j d 6$ consists of seven exons, with exon 4 occurring in two different variants $4 \mathrm{a}$ and $4 \mathrm{~b}$, respectively. At the protein level both new detected splice variants (transcripts 2 and 3 in Figure 3A) affect the C-terminal part of the Jmjd6 protein. In comparison to the main transcript annotated in Ensembl (transcript 1 in Figure 3A), either a stretch of 9 amino acids starting at position 302 is exchanged against 31 other residues (transcript $\mathrm{Mm} 3$ in Figure 3C) or a premature stop codon is intro- 

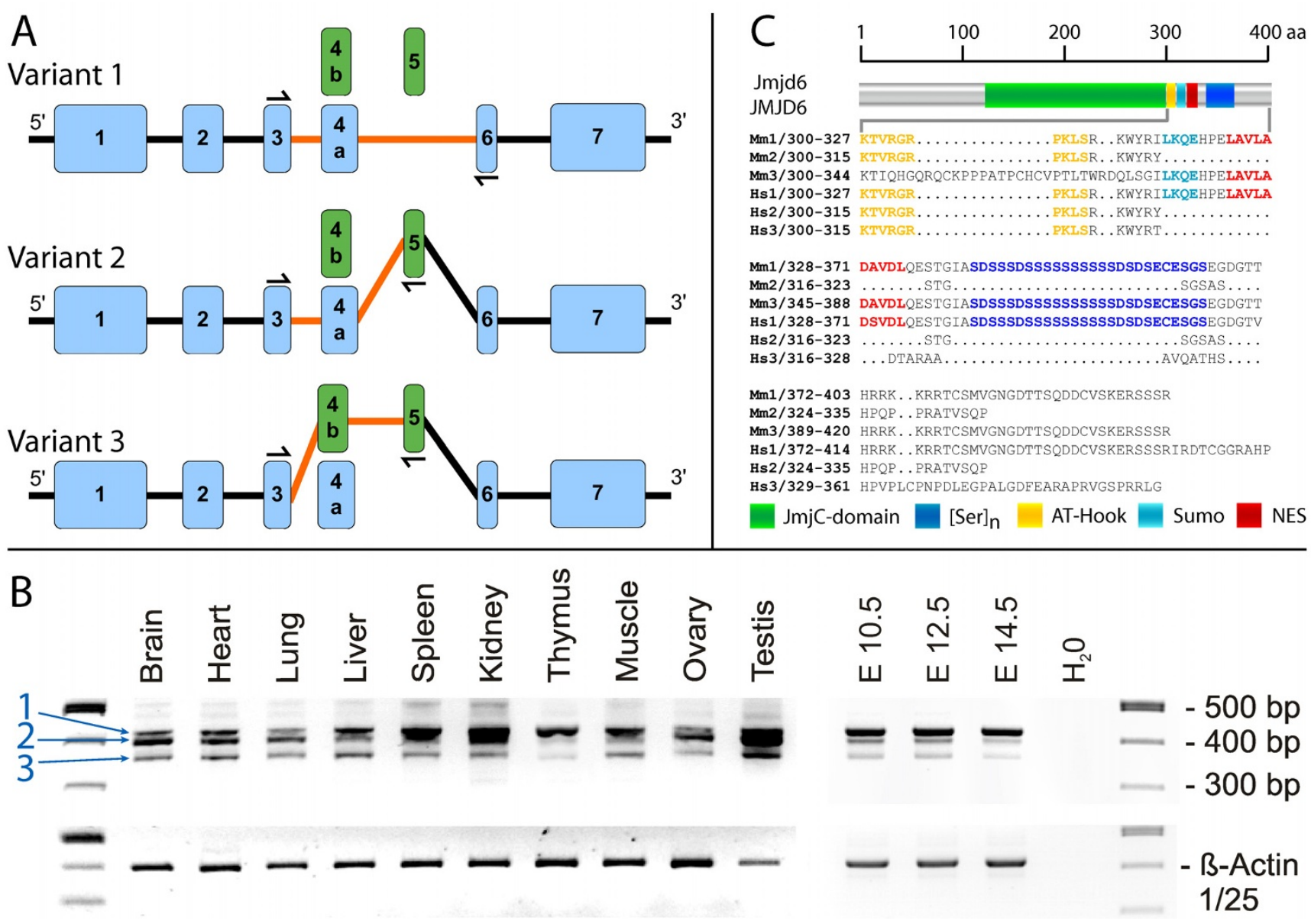

\section{Figure 3}

Identification and characterisation of new splice variants of Jmjd6. (A) Two additional putative exons (green boxes) were identified in addition to the transcript annotated in Ensembl (variant I) by screening all public EST databases. Alternative splicing using these two exons results in the generation of two additional transcripts of Jmjd6 (variants 2 and 3 , respectively). Half arrows and orange lines in the schematic presentation of the transcripts highlight the combination of exons detected by RT-PCR. Numbering of Jmjd6 exons includes the additional identified exon variants $4 \mathrm{~b}$ and 5. (B) Expression analysis of alternative Jmjd6 transcripts in adult mouse organs and embryonic stages (E) using RT-PCR confirmed the presence of all three transcripts at different mRNA expression levels in the analysed samples. Half arrows in $(A)$ indicate the position of the PCR primers used for the amplification. Blue numbers and arrows in (B) point to PCR fragments amplified from splice variants I to 3. Amplification of the housekeeping $\beta$-actin gene was used as a loading control. (C) The effects of the different splicing events on the C-termini are shown in the gapped alignment from amino acid 300 onwards. The full length human JMJD6 and murine Jmjd6 proteins are represented in the upper part of the figure. Nearly all novel splice variants identified in the mouse (Mm 2) and in humans (Hs 2-3) are truncated at the C-terminus in comparison to splice variant I (Mm I and Hs I) and are predicted to contain no poly-serine stretch $\left([\mathrm{Ser}]_{n}\right)$. In contrast, the JmjC domain is not affected (green box). Predicted AT-hook domain, sumoylation recognition site (Sumo), and nuclear export signals (NES) are annotated as depicted at the bottom.

duced resulting in a truncated protein of 314 amino acids (transcript Mm2 in Figure 3C). Using the CBS-prediction service, we identified in this region of the protein a putative sumoylation site (probability score $92 \%$ ) and a nuclear export signal (probability score 96\%) using NetNES. Furthermore, this region contains the previously described putative AT-hook domain [57] and the poly-serine stretch domain $([69,70]$ Figure 3C). All these potential interaction sites are not present in the protein encoded by transcript Mm2. Transcript Mm3 differs in comparison with the main transcript $(\mathrm{Mm} 1)$ in a stretch of 31 amino replacing the putative AT-hook containing region (Figure 3C).

Based on the identification of the highly conserved sequence coding for the new exon 5 in all mammalian species (Figure 2 and data not shown), we performed a similar e2g and Sim4 analysis using the human JMJD6 
locus and human EST database entries. This analysis confirmed the presence of exon 5 containing JMJD6 transcripts in humans and also the presence of two different variants of exon 4 in the human genome. Using again RTPCR, we validated the expression of these transcripts in vivo. Similar to the mouse we found expression of the three transcripts in 10 human tissues [see Additional file 1]. These, as well as the mouse alternative transcripts, were further validated and confirmed by sequencing (Accession numbers EF527404 for Mm2 and EF527405 for Mm3 for mouse Jmjd6 and EF527406 for Hs2 and EF527407 for Hs3 for human JMJD6 transcript variants, respectively). In comparison to the new identified mouse transcript variants, both human transcript variants are predicted to generate truncated proteins. Neither of these predicted proteins appear to encode a nuclear export signal, sumoylation site, AT hook domain or poly-serine stretch at the C-terminal part of the protein (Fig. 3C). As an insertion of premature translation termination codons might result in nonsense-mediated decay (NMD) of the corresponding mRNA [71], we analysed if the mouse transcript variant 2 and human splicing variants 2 and 3 might be subjected to degradation by the NMD pathway. Using RTPCR assays for each of these variants we found that this is not the case. Primers located in the new introduced exons and at the 3'-end of each transcript yielded products of the expected size, indicating that these variants are indeed expressed and most likely functional [see Additional file 2]. The human and mouse splice variants seem to be expressed at different mRNA levels depending on the tissue analyzed (see Figure 3 and [see Additional file 2]), with mouse splice variant $\mathrm{Mm} 1$ expressed in higher amounts in the developing embryo (Figure 3). Interestingly, an e2g and Sim4 analysis performed in non-mammalian species did not result in the recognition of mouse and human related Jmjd6 splice variants in these organisms (data not shown).

To analyse the conserved, putative bi-directional promoter element in between Jmjd6 and 1110005A03Rik, we tried to identify potential regulatory motifs in the genomic sequence encompassing the intergenic region, the 5'-UTRs and the first exons of both genes. We isolated a 840 bp fragment covering the genomic region between Jmjd6 and 1110005A03Rik (Figure 4A) that contained several predicted transcription factor binding sites based on a MatInspector analysis (data not shown). This fragment was further analysed using a promoter-less luciferase reporter gene assay. In comparison to controls, the selected genomic fragment clearly possesses transcriptional promoter activity when transfected into primary mouse embryonic fibroblast cells (MEFs) and various cell lines (Figure 4B and data not shown). Moreover, this transcription activating activity was found to be orientation dependent. In the forward strand orientation (which con- trols in vivo 1110005A03Rik expression according to the genomic annotation in Ensembl) the fragment showed only half of the activity as compared to the reverse strand orientation (Figure 4B), which controls expression of Jmjd6 in vivo. This demonstrated that the short intergenic region between Jmjd6 and 1110005A03Rik is capable to control basal expression of both genes, although this bidirectional transcriptional unit seems to activate both genes at different levels.

We then went on to investigate the consequences on Jmjd6, 1110005A03Rik and Sfrs2 expression of the different gene targeting strategies used to generate the three Jmjd6 mouse knockout alleles. In all three Jmjd6 mutant mice (JmjdG $\sigma^{\mathrm{m}} \mathrm{Flv}$, generated by the Flavell laboratory;

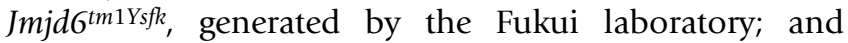
$J m j d 6^{t m 1 G b f}$, generated in our laboratory) the first two or three exons of the gene have been deleted. However, an alignment of restriction enzyme sites used to construct the different gene targeting vectors reveals that in JmjdG $\sigma^{\mathrm{m}} 1 \mathrm{Fl} v$ and JmjdG $\sigma^{\text {tm } 1 \text { Ysfk }}$ mice, in contrast to the JmjdG ${ }^{\text {tm1Gbf }}$ mouse line, exon 1 of 1110005A03Rik was deleted in addition [see Figure A in Additional file 3]. This was confirmed when we analysed the expression of all three genes in homozygous embryos harvested from the three mutant

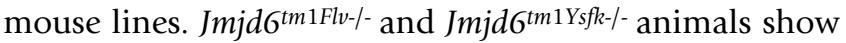
no expression of 1110005A03Rik and JmjdG, while the expression of $S f r s 2$ remains unaffected by the gene targeting. In Jmjd6 ${ }^{\text {tm } 1 G b f}$ homozygous embryos 1110005A03Rik, and Sfrs 2 are expressed comparable to wild type levels [see Figure B in Additional file 3] and as previously shown [54], only the expression of Jmjd6 is ablated in this mouse mutant line. This was further supported by semiquantitative expression analysis of 1110005A03Rik in JmjdG tm1Gbf-/ - embryos and primary fetal liver-derived macrophage cultures. RT-PCR analysis of serial diluted cDNA shows equal expression of 1110005A03Rik in JmjdG $\mathrm{tm}^{\mathrm{m}} \mathrm{Gbf}$ animals in comparison to wildtype controls in all analysed samples [see Figure C in Additional file 3]. Thus, the JmjdG ${ }^{t m} 1$ Flv and

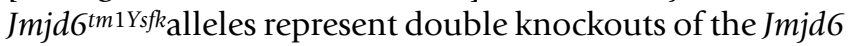
and 1110005A03Rik genes, while the JmjdG tm1Gbf allele ablates only Jmjd6 gene expression.

\section{Phylogenetic analysis of the Jmjd6 protein}

Following the identification of Jmjd6 orthologues in 30 of the 34 species annotated in Ensembl (Table 1), which implied a broad distribution of Jmjd6 during evolution, we continued to search for additional homologous proteins in other species. To identify further candidate proteins, we performed a comprehensive database (nr) search using the blastp algorithm and the $\mathrm{N}$-terminal part encompassing amino acids 53 to 152 of the mouse Jmjd6 protein. For the search strategy, we explicitly excluded the highly conserved JmjC domain to reduce the number of false positive hits representing Jmjd6-unrelated JmjC 


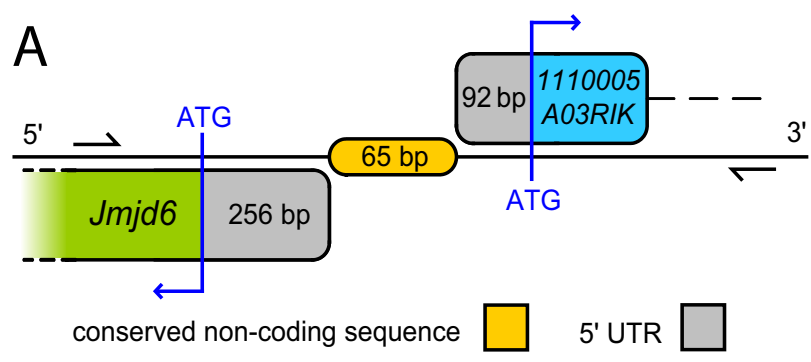

B

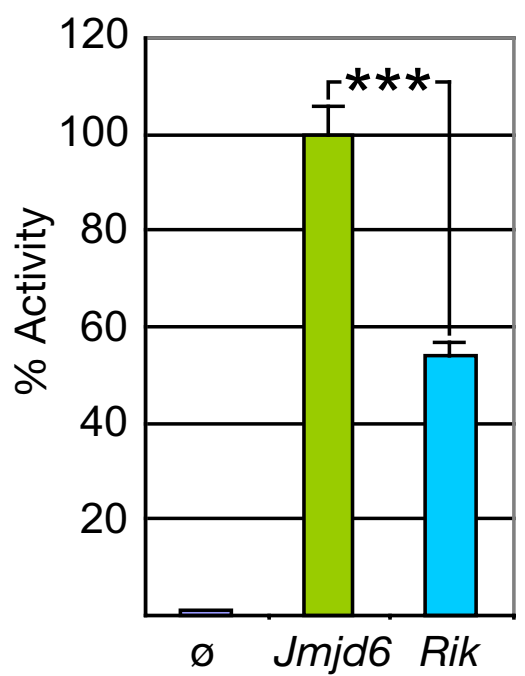

Figure 4

Identification of the Jmjd6 - I / /0005A03Rik bi-directional transcriptional unit. $(A)$ Schematic overview of the 65 bp non-coding and non-overlapping intergenic region between the murine Jmjd6 and I I I 0005 A03Rik (Rik) genes. The conserved sequence element identified using PipMaker in Figure 2 is shown in orange. $5^{\prime}$-untranslated regions (UTRs) are indicated in grey, and first exon coding sequences of the Jmjd6 gene in green and of the I / 10005 A03Rik gene in blue, respectively. The region indicated by two half arrows was used for promoter activity assays presented in (B). It was amplified and cloned to yield an 840 bp test fragment (B) This putative bi-directional promoter fragment was used in a luciferase reporter gene assay to measure transcriptional activity. Basal promoter activity was found for both transcriptional orientations compared to a promoterless control (pGL3) vector. The 5'-3' orientation of the fragment (I I 10005A03Rik orientation, Rik) yielded only half of the transcriptional activity as it was measured for the $3^{\prime}-5^{\prime}$ orientation (Jmjd6 orientation, Jmjd6). The values reported for the transfection experiments are means \pm standard deviation of three independent triplicate experiments. $P$ value was determined using Student's t-test. $* * * P<0.001$.

domain containing proteins. To ascertain that we select for the subsequent analysis in each species the sequence with the highest homology to Jmjd6, we used the "recip- rocal best BLAST hit" criterion [72]. The obtained fulllength database entries were used for a Mus musculusrestricted blastp-search of the nr database and for each species only those sequences matching Jmjd6 as the best BLAST hit criterion were retained for the analysis. As some sequences are only available from early stage annotation projects (low-sequence coverage genomes, [73]), we selected 54 out of 62 sequences due to quality measures as a working package for the calculation of a multiple sequence alignment by employing ClustalW. Following five iterations of re-alignment of the protein sequences the multiple sequence alignment was shaded with respect to amino acid identity/similarity and a protein sequence logo was generated and adjusted to the background frequency of each amino acid in the alignment. In addition, predicted secondary structure elements and SMART domains of the mouse Jmjd6 protein were added (Figure $5)$. The retrieved reciprocal best BLAST hits from the different species and the quality of the multiple sequence alignment allowed us to confirm the high homology of these 54 proteins to the murine Jmjd6 protein (Figure 5). Many of those are with no doubt true orthologous proteins of the mouse Jmjd6. These are found in the mammalia, aves, amphibia, actinopterygii, urchordata, echinodermata, insecta, nematoda, platyhelminthes, and cnideria and demonstrate the remarkable conservation of the Jmjd6 gene during evolution (Figure 5 and [see Additional file 4]) as previously also noted by others $([56,57])$. However, some proteins identified in plants, protista, fungi differ from the "JmjC only" domain structure characteristic for the Jmjd6 proteins of animalia. For example, some of them contain nearly five times more amino acids or have in addition to the JmjC-domain single F-box domains [see Additional files 4 and 9]. Nevertheless, they are the closest relatives to the mouse Jmjd 6 as defined by the best reciprocal BLAST hit criterion. Thus, it is difficult to decide if these proteins might be true orthologs of the animalia Jmjd6 proteins. If they are, it could mean that additional protein domains have been lost from an ancient Jmjd6 protein during evolution in the animalia lineage.

To explore potentially important residues for Jmjd 6 function we compared the degree of sequence conservation across the aligned sequences and included in the alignment predicted tertiary structure elements of the protein. First, our analysis showed the presence of a 2-His-1-carboxylate facial triad $(\mathrm{HxDxnH})$, as a common structural motif among non-heme iron(II) enzymes, present in all identified Jmjd6 proteins (Figure 5), suggesting an evolutionary conserved enzymatic function of Jmjd6. We could identify within the predicted JmjC domain eight $\beta$-strands (I-VIII) that have been shown to form the characteristic jelly-roll topology in other structurally resolved JmjC domain containing proteins [6]. The amino acids encom- 

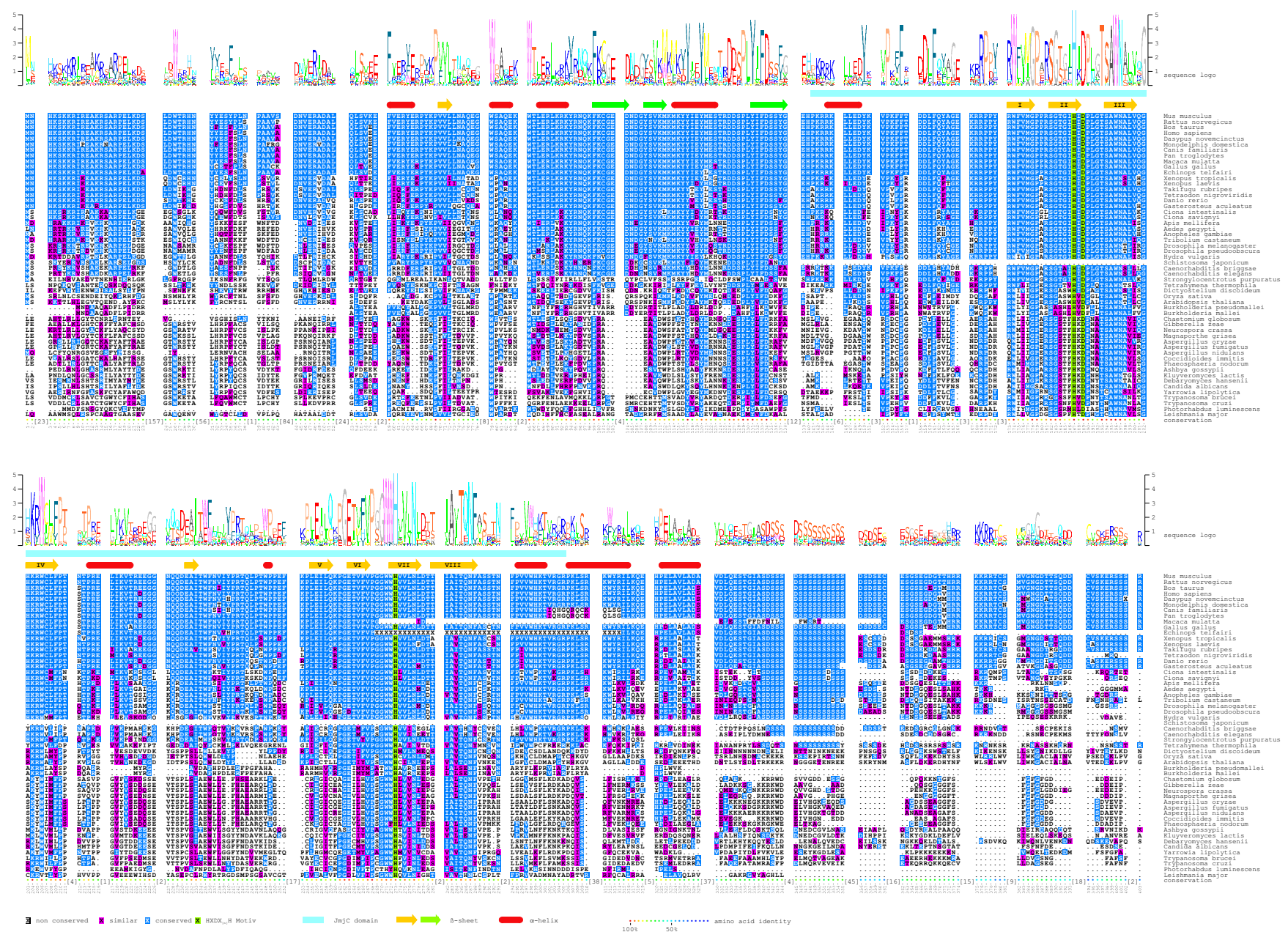

\section{Figure 5}

Multiple sequence alignment of homologous Jmjd6 proteins. The sequences of 54 homologous Jmjd6 proteins were used for the calculation of a multiple sequence alignment (ClustalW). The numbering of the residues is according to the mouse protein sequence. Identical residues were shaded in blue and similar residues in magenta. Green shading indicates the predicted catalytic residues of the $\mathrm{HxDx}{ }_{\mathrm{n}} \mathrm{H}$ facial. Sequence insertions in individual species outside of the conserved core regions of the mouse Jmjd6 protein were masked out and the maximum numbers of amino acids in these insertions are indicated [n]. Based on the sequence conservation, a frequency corrected sequence logo was calculated (shown above the alignment) and the mouse sequence conservation was highlighted in colour dots according to their degree of conservation (shown as colour code in percent identity below the alignment). The JmjC domain (light blue bar) and additional identified secondary structure elements (red cylinders: $\alpha$-helices; yellow and green arrows: $\beta$-strands) were annotated to the sequence as indicated at the bottom and the $\beta$-strands forming the jelly-roll of the JmjC domain were numbered (I-VIII). Green arrows represent the $\beta$-sheet that extends the major sheet outside of the jelly-roll.

passing these $\beta$-strands are highly conserved in all proteins. $50 \%$ of all $\beta$-strands forming amino acids are identical in at least $85 \%$ of the analysed species suggesting the spatial and functional conservation of the JmjCdomain in all analysed proteins. The only exception we found is in between $\beta$-strands IV and V. At this position we found an insertion of sequences of variable amino acid conservation among species and of varying length as it has been previously described for other JmjC domain containing proteins $[6,22,53]$. Second, within the C-terminal region of the Jmjd 6 protein preceding the last $\beta$-strands of the recognised jelly-roll the degree of sequence conservation is decreased. In contrast to the metazoan species showing a high degree of sequence identity, all other species display a significantly reduced similarity in this region. Furthermore, only the metazoan species have a poly-serine stretch in the C-terminal region of the Jmjd6 protein (Figure 5 and [70]). We could not identify any comparable sequence motif in the other species but looking for chemical properties of amino acids present at this 
position in proteins of non-metazoan species we found an enrichment of polar amino acids in this part of the protein (data not shown). This suggests at least a conservation and fortification of physico-chemical properties in this region during evolution. Third, the part $\mathrm{N}$-terminal of the JmjC domain shows in comparison to the C-terminus a higher degree of conservation. We found that $20 \%$ of the amino acids $59-140$ are identical in at least $90 \%$ of the analysed proteins (Figure 5). In addition, we identified one highly conserved and three less conserved $\beta$-strands in this region. These findings suggest the presence of an important structural element of the Jmjd6 protein in close vicinity to the predicted JmjC domain (see below).

\section{Comparative modelling and analysis of the conserved putative catalytic core domain of Jmjd6}

Due to the fact that up to now no crystal structure of Jmjd6 is available, we decided to perform a homology/ comparative modelling of the three-dimensional structure of the protein. The multiple sequence alignment of Jmjd6 homologous proteins (amino acids 44-329; Figure 5) was used for pairwise comparison of profile hidden Markov models (HMMs). This generated an alignment of the protein sequence that can be modelled with known related structures. This first step showed a significant probability $(=95 \%)$ of structural similarity of Jmjd6 to the three structurally resolved 2OG- and $\mathrm{Fe}(\mathrm{II})$-dependent dioxygenases Hiflan (PDB entry $1 \mathrm{~h} 2 \mathrm{k}$ ), putative asparaginyl hydroxylase - 2636534 from Bacillus subtilis (PDB entry 1 vrb) and Jmjd2a (PDB entry 2gp5), respectively. The alignment generated by HHpred [74] was finally prepared to create a structural model and MODELLER [75] calculated this comparative model of the Jmjd6 protein based on the identified similarities containing all non-hydrogen atoms (Figure 6). The comparative modelling of Jmjd6 predicted that the Jmjd6 fold is a mixed alpha-beta structure composed of a major and a minor $\beta$-sheet surrounded by several $\alpha$-helices. The structure showed the presence of the 2OG- and Fe(II)-dependent dioxygenase typical doublestranded $\beta$-helix (DSBH) motif as the protein core. It consists of two anti-parallel $\beta$-sheets composed of eight $\beta$ strands. The major sheet is defined by $\beta$-strands I, VIII, III, the minor sheet by $\beta$-strands VI and II, VII, IV, and V (Figure 6 and [see Additional file 7]), which form a jelly-roll topology. In addition, we recognised in the model three $\beta$ strands extending the core DSBH, resulting in a sevenstranded major $\beta$-sheet (green arrows in Figure 5 and orange arrows in Figure 6A). Interestingly, these additional strands were found to be paired anti-parallel to each other and to the major $\beta$-sheet.

Following iterative backbone annealing based on the structure of Hif1an (PDB entry $1 \mathrm{~h} 2 \mathrm{k}$ ), $2 \mathrm{OG}$ and Fe(II) were added to the 3D model of Jmjd6. After energy minimisation utilising the SwissPDB Viewer, the predicted active site of the Jmjd6-Fe(II)-2OG complex was analysed in detail. Here, we found the proper positioning of the 2His-1-carboxylate facial triad chelating the iron atom in a manner similar to other 2OG- and Fe(II)-dependent dioxygenases (Figure 6B). The HxD motif (His 187 and Asp 189 ) is predicted to be located just after the sequence of DSBH $\beta$-strand II and the second $\mathrm{Fe}(\mathrm{II})$-binding histidine (His 273) is located on DSBH $\beta$-strand VII. The observation of $\mathrm{Fe}(\mathrm{II})$ coordination by these three residues confirms their initially suggested assignment based on the primary sequence analysis of the multiple sequence alignment of Jmjd6 proteins (Figure 5). In addition, we could identify in the model several residues within the putative active core of Jmjd6 capable of positioning the co-substrate 2OG in the cavity formed by the interior of the DSBH. The proper positioning of 2OG relative to the $\mathrm{Fe}$ (II) seems to be achieved by an interaction of several residues of the active core that are close enough to the hydrogen-bond with the 2OG. Among these residues we predict Asn197 and Trp174 to interact with the 1-carboxylate oxygen of $2 \mathrm{OG}$ that is not ligated to the $\mathrm{Fe}(\mathrm{II})$. Asn 197 and Trp174 are stabilised by Thr285. Interestingly, in Jmjd6, like in Hif1an and Jmjd2a, a lysine (Lys204, from DSBH $\beta$-strand IV) could be important for the binding of the C5'-carboxylate of 2OG. Most of the other structurally resolved 2OG- and Fe(II)-dependent dioxygenases have instead of the threonine 285 an arginine at this position, which is part of a RXS motif within the DSBH $\beta$-strand VIII $[76,77]$. Thus, Jmjd6 has in contrast to other 2OG-dependent dioxygenases no tripeptide RXS motif (Figure 5). In addition to these major 2OG interacting residues in Jmjd6, we could recognise additional residues (Phe133 and Val275) for putative hydrophobic interactions in the active core of the protein.

Beside the high probability of the Jmjd6 structure deduced from the comparative modelling approach, we searched for an additional, independent validation of this prediction. To achieve this, we decided to combine phylogenetic sequence analysis data and our comparative modelling data to proof the reliability of the Jmjd6 protein structure prediction. Accordingly, the amino acid identity across all 54 analysed species was translated into an indicative colour code ranging from dark red, representing amino acid identity across all species, to dark blue for highly variable/non-conserved residues (Figure 5). This colour code was applied to the calculated Jmjd6 structure and the individual amino acids were coloured according to their conservation (Figure 7). This computational approach demonstrated a strong conservation of the predicted Jmjd6 catalytic triad during evolution with $100 \%$ sequence identity of the HxD motif in all analysed 54 species (Figure 7A). In addition, residues that are predicted to bind 2-OG (Asn197, Thr285, Trp174, Lys204, and Thr187) are also highly conserved (Figure 7B), 


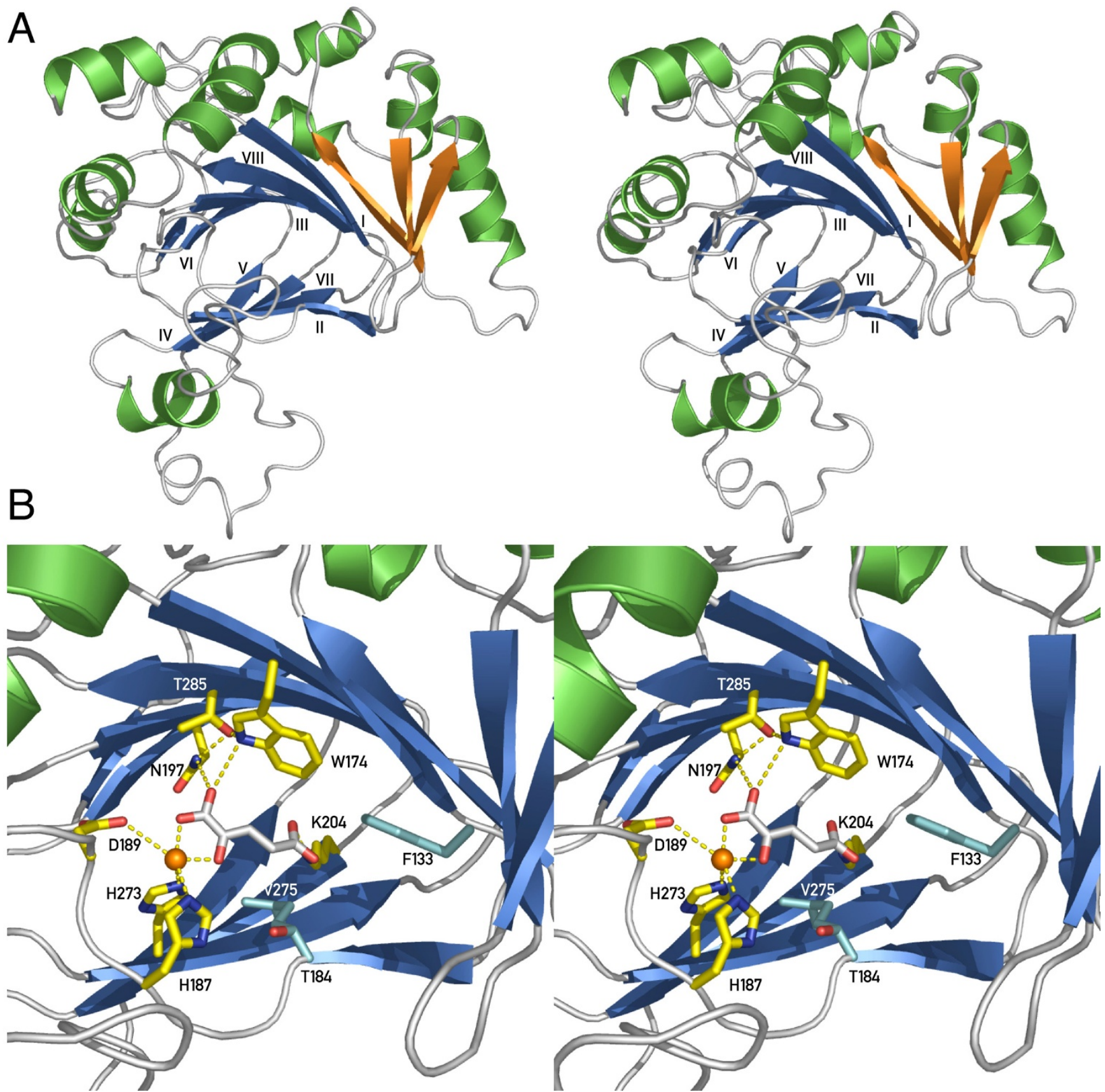

Figure 6

Predicted structure of Jmjd6 based on comparative modelling (stereoview). Jmjd6 sequence alignments (Fig. 5) and HHpred were used to model Jmjd6 utilising the PDB structures of Hiflan (Ih2k), Putative asparaginyl hydroxylase -2636534 ( $\underline{\text { vrb }}$ ) and Jmjd2a (2gp5), respectively. (A) Stereoview of the predicted structure of Jmjd6 presented as a ribbon model. The conserved eight-stranded DSBH core found in all $\mathrm{Fe}(\mathrm{II})$ and 2-oxoglutarate (2OG)-dependent dioxygenases is coloured in blue. Additional $\beta$-strands attached to the major $\beta$-sheet are shown in orange. Helices are depicted in green. (B) View of the predicted active site of the Jmjd6-Fe(II)-2OG complex showing coordination of Fe(II) to 2OG, His 187, Asp I89, and His273. 2OG also ligates to Trp 174, Asn 197 and Lys204 with Thr285 stabilising Asn 197 and Trp 174. Additional important residues for putative interactions are shown in cyan and include hydrophobic interactions from Phel33 and Val275 as well as Thr 184, which is involved in 2OG-binding in Hiflan. Interacting residues along with the 2OG co-substrate are shown as stick presentations, putative $\mathrm{H}$-bond interactions as dotted lines. $\mathrm{Fe}(\mathrm{II})$ is depicted as an orange ball. 
strongly suggesting that Jmjd6 is indeed possessing a 2OG-dependent dioxygenase function. Furthermore, a surface model of the protein with annotated sequence conservation shows that the putative active centre is located in a deep and narrow pocket [see Additional file 5]. Interestingly, the surface protein model also shows conservation of an exposed N-terminal region between $\beta$ strands 2 and 3 of the major $\beta$-sheet that is extending the DSBH core indicating that these residues might be important for interaction with other proteins [see Additional file 5]. In contrast to the sequence conservation observed in the predicted catalytic core and the exposed N-terminal major $\beta$-sheet, other regions of the protein have been much less conserved during evolution. Thus, the major selective pressure seems to have been on the catalytic function of the protein, while other areas of the protein retained flexibility in their functional design.

\section{Integration of Jmjd6 into the JmjC domain containing protein family}

By using the computational pipeline described in Figure 1 , we extracted a non-redundant set of $313 \mathrm{JmjC} / \mathrm{DSBH}$ domain-containing proteins from the Ensembl, Interpro, Uniprot and SMART databases. We selected these proteins from human and eight model organisms (mouse, zebrafish, pufferfish, tetraodon, nematode, fruitfly and yeast) based on the sequence homology of their JmjC domain to the mouse Jmjd6 and on the quality of the available sequence. Because genome-sequencing projects of many species are still ongoing, we had to exclude JmjC domain encoding genes from some species with lowsequence coverage genomes that did not match our quality criteria. Nevertheless, our comprehensive catalogue included $61 \mathrm{JmjC} / \mathrm{DSBH}$-domain containing proteins from Homo sapiens, 57 from Mus musculus, 49 from Danio rerio, 53 from Tetraodon (T. nigroviridis and T. rubripes), 45 from Drosophila melanogaster, 27 from Caenorhabditis elegans, 6 from Saccharomyces cerevisae, and 10 from Schizosaccharomyces pombe [see Additional file 8]. The catalogue contains all classes of JmjC domain containing proteins that have been described so far, including "JmjC domain only" proteins. To classify these proteins into evolutionary subgroups and to investigate their evolutionary relationship we analysed the selected sequences. A multiple sequence alignment and subsequent bootstrapping analysis was carried out using ClustalW (1000 bootstrap trials, 111 seeds) and PHYLIP. Based on the similarity of the JmjC domain, the phylogenetic analysis revealed 25 evolutionary conserved subgroups [see Additional file 6]. These subgroups are in general accordance to previous studies, which reported the classification of $44 \mathrm{JmjC}$ domain containing proteins into 4 related clusters [5] and of $98 \mathrm{JmjC}$ domain containing proteins into 7 subfamilies [4]. Although, our analysis shows that especially the previously classified subgroup of "JmjC domain only family"
[4] splits into 8 further subgroups based on the sequence divergence of their JmjC domain (1110034B05Rik family, Hif1an, Hspbap1, Pla2g4b, Jmjd5, Jmjd4, Jmjd6, JFP6like, and No66/Mina53 subgroups). As previously described [4,5], these proteins have beside their JmjC domain no other recognisable protein domain but they are clearly functionally distinct from each other regarding reported protein interaction partners, cellular localisation and physiological functions. For example, Hif1an is an asparagine protein hydroxylase for the HIF1 $\alpha$ transcription factor, Hspbap1 is a cytosolic protein that associates with heat shock protein $27 \mathrm{kD}$, and Jmjd6, Mina53, No66 are distinct nuclear proteins with as yet unknown molecular functions. In addition to these groups, we included in our analysis the AlkB, PH4/Phy-3, Ogfod2, Ogfod1/Tpa1, Plod 1-3, Lepre1/Leprel 1-2, 1110031I02Rik, and Egln13/Egl-9/Hph subgroups that were not analysed before together with the recently identified JmjC domain containing histone demethylase proteins [4]. Interestingly, we found that all subgroups could be classified independent of additional domain architectural features of the analysed proteins. As previously reported $[4,5]$, the domain compositions of individual proteins classified into a given subgroup varied, indicating that neither their enzymatic specificity nor their functional relationships can be predicted solely based on the analysis of primary protein sequences. This is further supported by the identification of certain members within subgroups that obviously can not possess enzymatic functions as 2OG-dependent dioxygenases due to sequence changes within their predicted $\mathrm{Fe}(\mathrm{II})$ and/or 2OG-binding sites. For example, the Hairless protein within the Jmjd1/Hairless subgroup, the Epe1 protein in the Jhd 1 subgroup and the whole Jarid 2 protein subfamily contain substitutions in these binding sites indicating that these proteins are unlikely to be functional enzymes ([4] and Figure 8).

Interestingly, in some subgroups we observed a huge expansion in the number of family members. The $\mathrm{P} 4 \mathrm{H} /$ Phy-3 family of prolyl-4-hydroxylases contained 22 proteins from Drosophila melanogaster. The $\mathrm{P} 4 \mathrm{H} / \mathrm{Phy}-3 \mathrm{sub}-$ group compromises prolyl-4-hydroxylases involved in collagen biosynthesis or hypoxia sensing. Currently, it is unknown why especially the fly has so many members in this protein family. Another example is the AlkB subfamily of DNA and RNA demethylating enzymes. This group contains 45 proteins that split into the Alkbh1 to Alkbh8 protein family members. Apart from Alkbh1 to Alkbh3 that are known to be DNA/RNA repair enzymes $[52,78,79]$ the exact biochemical activity of most of the other subfamily members has still to be determined.

Following phylogenetic analysis and tree generation a subtree encompassing the Jmjd6 protein was extracted for further analysis (Figure 8). By combining the information 


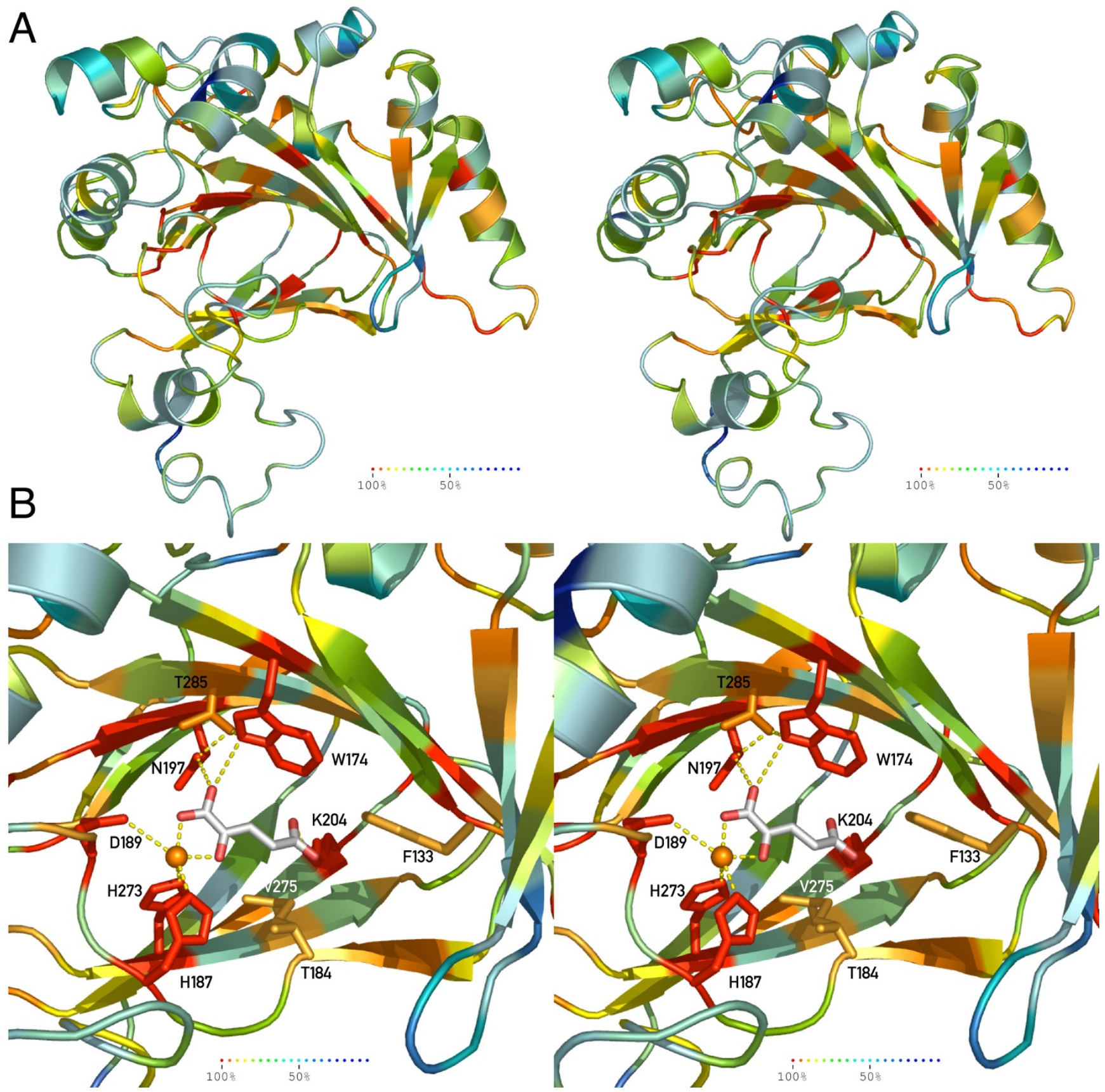

Figure 7

Evolutional conservation of critical residues in Jmjd6 (stereoview). (A) Ribbon presentation of the predicted Jmjd6 structure is coloured based on the sequence homology observed in 54 Jmjd6 homologous proteins (Fig. 5). (B) Annotation of the sequence identity onto the catalytic domain demonstrated the conservation of the critical residues within the active site of the Jmjd6-Fe(II)-2OG complex. Residues were shaded according to the heat map with dark red representing total conservation of residues (100\% sequence identity across 54 analysed species), and blue colour representing $50 \%$ sequence identity.

from the phylogenomic analysis with the annotation of protein subfamilies it is evident that Jmjd4 is closely related to Jmjd6 regarding sequence similarity. The JmjC domain of both proteins shows the highest sequence sim- ilarity among all 313 classified members (32\% sequence identity and $50 \%$ sequence similarity). This indicates that Jmjd4 is the closest paralogue of Jmjd6. 

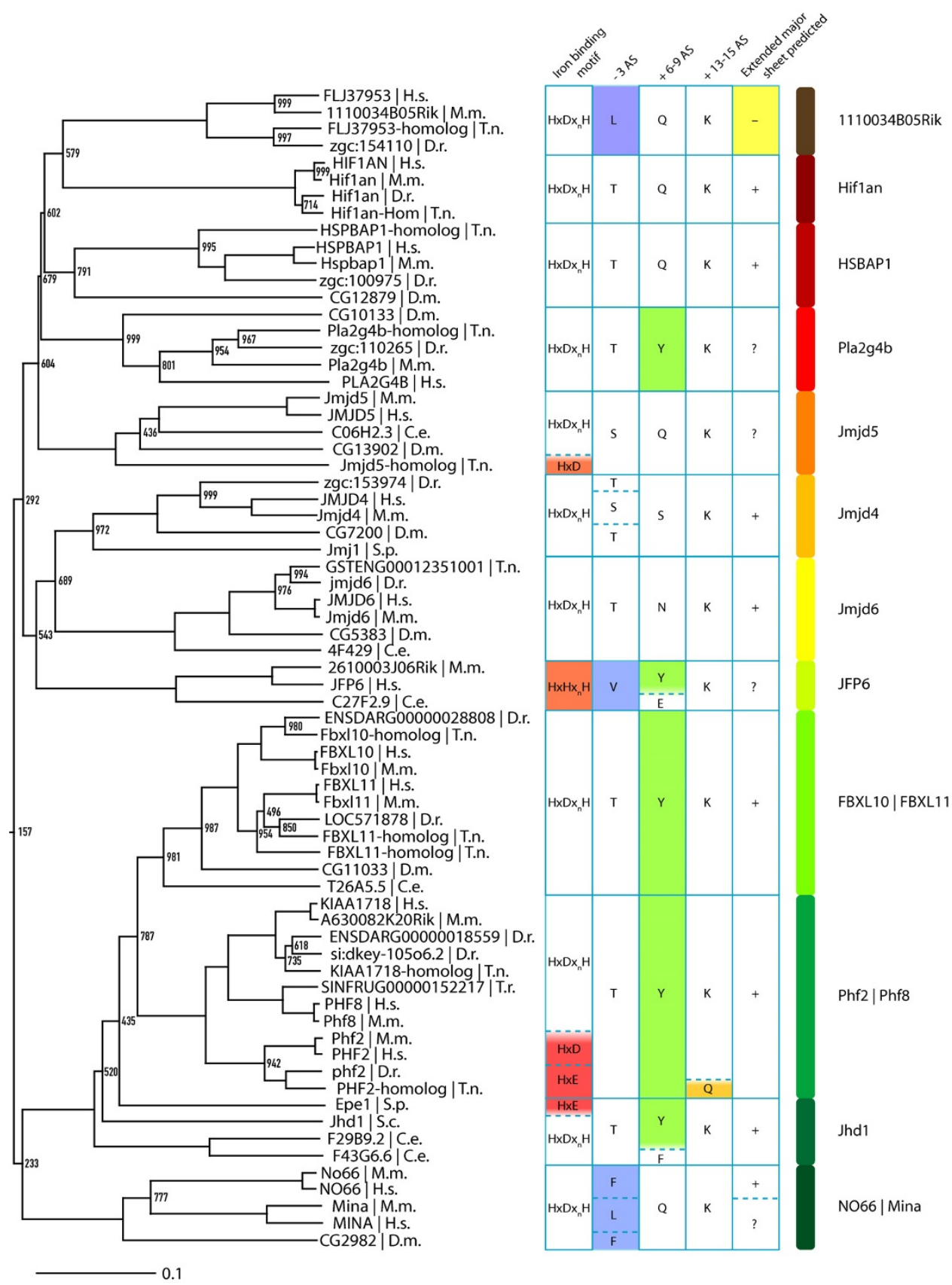

Figure 8

Phylogenetic relationship of Jmjd6 to other JmjC domain containing proteins. Detailed section of the phylogenetic tree of $313 \mathrm{JmjC}$ proteins that is presented [in additional file 6]. Shown are 12 out of 25 identified DSBH fold containing protein subgroups as indicated with protein family names on the right side. Scale bar represents the relative phylogenetic distance as determined with PHYLIP. Bootstrap values are shown for values $<1000$. The first column of the table shows the residues potentially involved in iron binding. The second, third and fourth columns show potentially important residues within the -3 , $+6-8$ and $+13-15$ regions in respect to the $\mathrm{Hx}(\mathrm{D} / \mathrm{E} / \mathrm{H})$ motif, respectively. Residues discussed in the text are highlighted in red, orange, blue, green and yellow. The fifth column shows the predicted ability to build an extended major sheet with three antiparallel $\beta$-strands as determined by Jpred. A "+" indicates the presence of three beta strands, a "?" two beta-strands and a "" no predicted beta-strands within a 50 aa window starting 80 aa before the first $\beta$-strand from the JmjC jelly-roll. H.s. $=$ Homo sapiens, M.m. = Mus musculus, D.r. = Danio rerio, T.n. = Tetraodon nigroviridis, T.r. = Takifugu rubripes, D.m. = Drosophila melanogaster, C.e. $=$ Caenorhabditis elegans, S.c. $=$ Saccharomyces cerevisiae, S.p. $=$ Schizosaccharomyces pombe. 
Moreover, a detailed sequence analysis of JmjC domain containing protein subfamilies that are related to the Jmjd6 and Jmjd4 subgroups revealed further insights into $\mathrm{Fe}$ (II) and 2OG coordinating mechanisms (Figure 8). The JFP6 subgroup has a HxHxH motif instead of the $\mathrm{Hx}(\mathrm{D} /$ E) $\mathrm{x}_{\mathrm{n}} \mathrm{H}$ motif that is commonly used by all other JmjC domain containing proteins for the coordination of $\mathrm{Fe}(\mathrm{II})$ (Figure 8, orange box). If this motif is compatible with an oxygenase activity, as one would expect, has to be investigated in the future. Furthermore, the Jmjd5 homologue of Tetraodon nigroviridis, all Phf2 proteins, and Epe1 have only a $\mathrm{Hx}(\mathrm{D} / \mathrm{E})$ motif (red box in Figure 8 ) and therefore lack the second histidine, which is known to be important for iron binding. Moreover, the T. nigroviridis Phf2 homologue lacks in addition the lysine that stabilises the C5'caboxylate of 2OG. Next to this motif, Tuskada et al. described residues in positions $-3,+6-8$ and $+13-15$, in respect to the HxD motif to be important for the catalytic function of histone demethylases [21]. In the -3 position, the 11100034B05Rik, JFP6 and No66/Mina subgroups have a nonpolar side chain present in contrast to the polar side chains of serine/threonine residues in other subgroups. In the $+6-8$ position, members of the Pla2g $4 \mathrm{~b}$, FBXL10/FBXL11, Phf2/Phf8, and individual members of the JFP6 and Jhd 1 subgroup have a conserved tyrosine. In Epe1, this tyrosine has been shown to be important for heterochromatin destabilisation, which is independent of an Epe1 histone demethylation activity $[21,46]$. Another interesting feature is the predicted presence of an extended major $\beta$-sheet in most of the JmjC domain only protein families encompassing the subtree (Figure 8).

\section{Discussion}

Here we report a systematic annotation and comparison of Jmjd6 homologous proteins across diverse phyla. With the recent description of the JmjC domain as a novel demethylase signature motif [21] and the discovery that many proteins containing JmjC domains are involved in reversing histone methylation marks $[4,80]$, this protein superfamily has attracted central interest in many different fields of biology. It has become quite clear that these enzymes have not only important functions in the epigenetic regulation of chromatin modification states but also in diverse other physiological contexts ranging from oxygen sensing to fatty acid metabolism and DNA repair [81]. However, for most of these proteins it has remained difficult to unravel their molecular function or to predict their enzymatic targets solely based on sequence comparisons and analysis of their JmjC domains. In the past, we have been interested in the "phosphatidylserine receptor" (now renamed into jumonji domain containing 6), a JmjC domain containing protein, which was initially described to function as a type II transmembrane receptor for the engulfment of apoptotic cells [56]. To compare the JmjC domain of Jmjd6 to the DSBH/JmjC domains of other members within this protein superfamily we designed a semiautomated sequence retrieval and analysis routine that allowed us to establish a catalogue of 313 $\mathrm{DSBH} /$ JmjC domain containing proteins in nine selected species. In addition, we analysed the Jmjd6 and neighbouring loci in vertebrates and investigated their modes of expression.

\section{Comparative analysis of the vertebrate Jmjd6 locus and immediate neighbouring loci}

With the ongoing genome sequencing projects in many different species and integrated efforts to annotate the functional content of fully sequenced genomes there is a need to complement existing automated genome annotations with a focused bioinformatics analysis of individual genes and gene families to make fully use of publicly available databases and resources. Such approaches might help to correct mistakes in automated annotation procedures and might add additional information to gene sets. This will finally lead to a more comprehensive and accurate annotation of gene functions.

We used the PipMaker alignment tool to analyse the patterns of sequence conservation across the mouse Jmjd6 and the neighbouring loci 1110005A03Rik and Sfrs2. To identify all protein coding exons as well as highly conserved non-coding sequence elements in this region we analysed genomic sequences of 22 different vertebrate species. This led to the identification of an up to now overseen additional Jmjd6 exon in mammals and to the detection of a conserved putative bi-directional promoter element in between the closely spaced Jmjd6 and $1110005 A 03 R i k$ genes. The re-annotated genomic region encompassing the Jmjd6, 1110005A03Rik and Sfrs2 loci was then the basis for a comparison of gene-targeting strategies that have been used to generate three different loss-of-function alleles of Jmjd6 in the mouse [54,62,63]. By aligning a map of restriction enzyme sites used for the construction of the different targeting vectors with the genomic region encompassing the three genes, we could show that in the Jmjd $\sigma^{\text {tm } 1 F l v}$ allele and in the JmjdG $\sigma^{\text {tm } 1 Y s f k}$ allele exon 1 of the 111005A03Rik gene as well as the conserved intergenic region in between Jmjd6 and 111005A03Rik was deleted upon homologous recombination in ES cells. An expression analysis of Jmjd6, 111005 A03Rik and Sfsr2 in the three different mouse alleles confirmed that Jmjd $\sigma^{\mathrm{tm} 1 F l v}$ and $J m j d \sigma^{\mathrm{tm} 1 Y^{\text {sfl }}}$ mutant mice are double knockouts of the Jmjd6 and 111005A03Rik genes. In contrast, the JmjdG ${ }^{\text {tm } 1 G b f}$ allele ablates only the expression of Jmjd6. Although all three independently generated knockout mice show to some degree overlapping phenotypes regarding defects during embryogenesis (e.g. disturbed eye development, brain malformations and defects in haematopoiesis) other controversial observations concerning the description of specific phenotypes 
have been reported $[54,62,63]$. Foremost, the capabilities of the mutant mouse lines to engulf apoptotic cells have been described differently. While Kunisaki et al. [63] and Li et al. [62] reported that their knockout mice showed impairment in the clearance of apoptotic cells during embryogenesis and defects in apoptotic cell phagocytosis assays, no such differences in phagocytosis competence

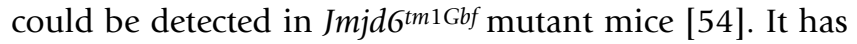
been suggested that differences in genetic backgrounds might account for these reported phenotypic variations [82-84]. However, in the light of the described results with JmjdG ${ }^{\text {tm } 1 F l v}$ and JmjdG ${ }^{\text {tm1 } 1 \text { ssf }}$ mutant mice being double knockouts of Jmjd6 and 111005A03Rik the situation might be far more complex. It could well be that loss-of 111005A03Rik function in JmjdG tmiFlv and JmjdG tm1 Ysfl knockout mice might contribute in addition to the Jmjd6deficiency to the phenotypic discrepancies observed. Future studies involving the generation and analysis of 111005A03Rik knockout mice will address this possibility.

Interestingly, the as yet functionally uncharacterised 111005A03Rik gene contains a predicted arginine protein methyltransferase domain. We demonstrate here, that the conserved sequence element in the short intergenic region between Jmjd6 and 111005A03Rik possess bi-directional promoter activities for both genes. Of note, the forward orientation of the fragment, which controls 111005A03Rik expression in vivo showed only half of the transcriptional activity as compared to the reverse orientation, which controls Jmjd6 expression in vivo. This indicates that both genes might compose a bi-directional transcriptional unit with opposing transcriptional and regulatory activities. This gives room for speculations. If 111005A03Rik has a function as a methyltransferase and the Jmjd6 protein possess demethylation activity as many other JmjC domain containing proteins are doing, both proteins might be involved in a common biochemical pathway targeting possibly a shared substrate. Interestingly, such counterbalancing principles of methylation and demethylation activity have been already suggested for other JmjC domain containing proteins [53]. For example, the Alkbh8 protein contains an amino-terminal JmjC domain, which might possess DNA demethylase activity that is fused to a predicted C-terminal methylase domain. This domain architecture is conserved in the homologous Alkbh8 proteins in Drosophila (CG17807) and C. elegans (C14B1.10) suggesting the selection of a controlled methylation/demethylation regulatory mechanism during the evolution of these proteins.

If Jmjd6 and 111005A03Rik also establish such counteracting functions needs to be addressed in the future. To proof such a hypothesis, the molecular function of both genes need first to be characterised. Nevertheless, the additional deletion of the 111005A03Rik gene in two of the three described Jmjd6 knockout mice might well explain differences reported for their apoptotic cell engulfment phenotypes. If 111005A03Rik is functioning as a methyltransferase in the control of transcriptional regulation, it is possible that the expression of other genes targeted or regulated by 111005A03Rik and/or Jmjd6 might influence phagocytosis of apoptotic cells. In such a scenario the described engulfment defects might be related to secondary effects caused by deregulation of genes involved in processes of phagocytosis or cellular differentiation of phagocytes.

Using PipMaker analysis based on sequence conservation we identified a before overseen exon in JmjdG loci of mammalian species. A subsequently undertaken e2g annotation of all available EST sequences to the Jmjd6/ JMJD6 genomic regions lead to the discovery of two new alternative transcripts in mice and humans. At the protein level the most distal C-terminal part of the Jmjd6 protein (last 100 amino acids) is affected in all new identified transcripts. Either truncated polypeptides are generated that miss the poly-serine stretch, the putative AT-hook domain, and the predicted sumoylation and nuclear export signal (NES) or a stretch of eight amino acid is exchanged with 31 other residues. In this second splice variant identified in mice (Mm3) the predicted sumoylation site, the NES and the poly-serine stretch are retained but the putative AT-hook domain is missing (Figure 3C). Our 3D structural model of the Jmjd6 protein indicates that the sumoylation site, the NES and the AT-hook motif might be accessible for interacting proteins. The AT-hook domain is known to mediate binding to DNA in highmobility group proteins [85]. Jmjd6 is a nuclear protein, which has been shown to contain five independently active nuclear localisation signals [58]. The NES might allow a shuttling of the protein from the nucleus to the cytoplasm. Likewise, the sumoylation site might be used for the regulation of interactions with other proteins.

A comparison of the Jmjd6 sequence with the resolved protein structure of the Hif1an/HIF1 $\alpha$ C-terminal transactivation domain co-complex indicates that interactions with other proteins might occur in the Jmjd6 C-terminal region [49]. Alternatively, at this site homo-di- or homomultimerization of Jmjd6 polypeptides might take place. For many JmjC/DSBH domain containing enzymes it is known that they can exist in various oligomeric forms. For example, Hif1an is a dimer in solution, germin forms a hexamer and HPPE a tetramer (for review see [6]). Indeed, very recently it could be demonstrated that Jmjd6 forms covalent multimers through homomeric polymerisation of the $50 \mathrm{kD}$ protein [61]. Nuclear aggregated Jmjd6 products with masses of 100,150, 200 and $250 \mathrm{kD}$ were detected after immunoprecipitation indicating that 
assembly of homomultimers might be important for the protein function [61]. Thus, the new alternative transcripts encoded by the Jmjd6 locus might possess a regulatory role by suppressing multimer formation or interactions with other proteins. Such possibilities need to be experimentally investigated in the future.

\section{Structural model of the Jmjd6 protein and evolutionary relationship to other JmjCIDSBH-domain containing proteins}

Recent crystallographic studies have helped to provide important insights in the structure and functions of JmjC domain containing proteins. More than ten structures of different members of this large oxygenase family have been solved demonstrating that the JmjC domain forms a characteristic DSBH structural fold composed of eight $\beta$ strands that build two $\beta$-sheets each containing four antiparallel $\beta$-strands [6]. The catalytic core of these metalloenzymes consists of a highly conserved $\mathrm{Hx}(\mathrm{E} / \mathrm{D}) \mathrm{x}_{\mathrm{n}} \mathrm{H}$ signature motif that supplies three chelating positions for the $\mathrm{Fe}(\mathrm{II})$ iron and two additional chelating positions are contributed by the substrate co-factor 2OG. Very often the binding sites for 2OG in the DSBH core are as well conserved among the members of this protein family. Due to high sequence similarity of Jmjd6 to other JmjC domain containing proteins [57], we have embarked on the modelling of the Jmjd6 3D-structure to gain further insights into possible protein functions. Our analysis shows that Jmjd6 is indeed most likely a DSBH-fold containing 2OGdependent dioxygenase enzyme, as previously first suggested by Cikala and colleagues [57]. All residues needed for $\mathrm{Fe}(\mathrm{II})$ coordination, 2OG co-substrate binding and oxidative catalysis were found to be located within the predicted DSBH domain of the protein model and are evolutionary preserved in all analysed Jmjd6 homologous proteins probably including those of non-metazoan species. We identified in all orthologous Jmjd6 proteins from metazoan species the characteristic $\mathrm{HxDx}_{[\mathrm{n}]} \mathrm{H}$ catalytical triad and our model predicts five conserved residues (Trp174, Thr184, Asn197, Lys204, and Thr285) as being important for interaction with a $20 \mathrm{OG}$ co-substrate. In addition, the extreme conservation of residues forming the eight $\beta$-strands of the DSBH domain suggests a spatial and functional conservation of the Jmjd6 JmjC domain during evolution. This makes it highly likely that all Jmjd6 proteins in the different animal kingdoms are indeed active 2OG-dependent dioxygenase enzymes. This might not be true for other members of the JmjC/DSBH fold superfamily of proteins. For example, the subfamily of JFP6-like proteins has a substitution in the Fe(II) coordinating residues changing the $\mathrm{Fe}(\mathrm{II})$ binding motif. Furthermore, hairless and Jarid2 proteins are most likely enzymatically inactive due to the missing $\mathrm{HxD}$ motifs in their DSBH protein cores [4]. This suggests that JmjC domains might possess additional functions that are independent of any enzymatic activity.

2OG-dependent dioxygenases are known to catalyse two types of posttranslational protein modifications. Described have been hydroxylation of the amino acid side chains of proline, asparagines, aspartic acid and tryptophan, and secondly demethylation reactions on lysine residues (for review see $[6,81]$ ). It has been proposed that also methylated arginines might be targets of JmjC domain containing proteins. In fact, most recently it has been suggested that Jmjd6 might possess an enzymatic activity as an arginine histone demethylase. It was reported that Jmjd6 is capable to catalyse the removal of di-methyl groups on histone H3 at arginine 2 (H3R2) and on histone 4 at arginine 3 (H4R3) [86]. Asymmetric dimethylation of H3R2 has been associated with repression of transcriptional activity through its enrichment in heterochromatic loci and inactive euchromatic genes $[87,88]$. Thus, Jmjd6 might have a function in gene activation by erasing repressive chromatin marks. But it is also not unlikely that additional, non-histone substrates are targeted by Jmjd6.

All types of posttranslational modifications catalysed by 2OG-dependent dioxygenases involve a common type of hydroxylation reaction. During the catalytic reaction, the splitting of molecular oxygen is coupled to the hydroxylation of the target substrate and the oxidative decarboxylation of $2 \mathrm{OG}$ to give succinate and $\mathrm{CO}_{2}$. In the case of demethylation, hydroxylation of a methyl group is followed immediately by deformylation producing formaldehyde as an additional reaction product. As noted before by others, it is impossible to predict the prime substrate of 2OG-dependent dioxygenase based on a primary sequence analysis of the JmjC domain [80,81]. Even JmjC domain containing enzymes of the same subfamily such as the JHDM1A and the JHDM2A histone demethylases that both recognise $\mathrm{H} 3 \mathrm{~K} 36$ methylation have very limited primary or secondary structure similarity [22]. This suggests a requirement for an extended substrate recognition mechanism that probably involves protein-protein interactions in larger protein complexes or for a necessity of very defined interaction sites in the catalytic core that are important for determining substrate selectivity [89]. This is further supported by a number of recent studies that implicated other methyl-dependent protein domains like Tudor, MBT, PHD fingers and chromo domains as reader domains in the recognition of the histone code [90-94]. Jmjd 6 contains a single JmjC domain and beside the predicted recognition sequences for nuclear in- and export, sumoylation and the AT-hook DNA-binding motif no other recognisable domain is present in the protein. Thus, the substrate specificity of Jmjd6 must be regulated by interaction with other proteins. 
What is quite clear is that the putative enzymatic function of Jmjd6 as a 2OG-dependent dioxygenase is linked to its important role in development and morphogenesis, at least in vertebrates. All mouse mutants conclusively point to an essential role of Jmjd6 during embryogenesis were the gene is involved in the differentiation of multiple tissues and organs. Also in zebrafish, the morpholino knockdown of the jmjd6 orthologue resulted in severe defects in somite, brain, heart and notochord development [95]. This may or may not involve secondary effects on apoptosis induction and cell corpse clearance. Unlikely is a direct involvement of jmjd6 in apoptotic cell recognition or engulfment. Given that many 2OG-dependent dioxygenases have been implicated in the regulation of multiple pathways, global effects on gene expression and metabolism are not surprising when their enzymatic functions get perturbed or ablated.

The function of Jmjd6 during development seems to be different in vertebrates and invertebrates. Inactivation of the orthologous genes in Drosophila and C. elegans were surprisingly not associated with embryonic lethality and no major developmental defects have been reported for the loss-of-function mutants [59,96]. Interestingly, mutant flies that lack $d P S R$ show inappropriate apoptosis during eye development [59]. Major phenotypes were observed in gain-of-function experiments in which the dPSR gene was overexpressed during development. Male transgenic flies that expressed $A P S R$ under ubiquitous promoters developed a genital rotation phenotype. $d P S R$ overexpression in the wing resulted in various wing blade fusion phenotypes and driving gene expression in the eye induced a small rough eye phenotype. Further genetic analysis pointed to an involvement of $d P S R$ in the head involution defective (hid) and c-Jun-NH2 terminal kinase pathways. These suggested that loss of $d P S R$ enhances apoptosis in the developing eye, while overexpression of $d P S R$ protects from apoptosis. Of note, the analysis of mutant flies did not reveal a role for $A P S R$ in the phagocytosis of apoptotic cells during development. Altogether, these observations support a function of $A P S R$ in cell survival and differentiation that might have parallels in the vertebrate system [59].

When we compared the protein sequences of vertebrate and invertebrate orthologous Jmjd6 proteins we found divergence in the $\mathrm{N}$ - and $\mathrm{C}$-terminal regions of the protein. Interestingly, the $\mathrm{C}$-terminal poly-serine stretch is either shorter or missing in the invertebrate species. Moreover, we could not identify an additional exon in the invertebrate orthologous Jmjd6 genes that would generate alternative transcripts with effects on the C-terminal part of the protein. Therefore, it could well be that differences in this region of the protein are linked in vertebrates with different physiological functions of Jmjd6 protein, possi- bly through interactions with other proteins. This is also supported by identification of proteins in plants, fungi and protista that show partial sequence homology to the animalia Jmjd6 protein (Figure 5 and [see Additional file $4])$. These were identified through sequence search routines focussing on a conserved $\mathrm{N}$-terminal sequence stretch outside of the highly conserved JmjC domain. Consequently, these proteins show in their N-terminal parts significant sequence homology to the mouse Jmjd6 protein, but are diverse to Jmjd6 in their C-terminal regions. Moreover, many of these proteins are longer then the animalia Jmjd6 proteins (up to 2.000 aa instead of around 400 aa for animalia Jmjd6 proteins) and contain often in addition to the JmjC domain a single F-box domain.

Thus, it is very likely that they possess different or additional functions than the animalia Jmjd6 proteins and a possible homology of these proteins to Jmjd6 should be treated with caution. As the reciprocal high similarity suggests a relation to Jmjd6, it might be possible to postulate an ancestral "Jmjd6" gene that encoded a larger protein with additional domains (like F-box domains), which then have been lost during evolution of JmdG genes in animalia. Alternatively, the evolution of Jmjd6 genes in animalia could have been an independent event and these genes are not related in any way to the proteins we identified in plants, fungi and protista. In the future it might be possible to consolidate on one hypothesis, given that more functional data about these hypothetical proteins are being gathered and higher quality sequence information becomes available for phylogenetic analysis.

\section{Conclusion}

Taken together, our integrated bioinformatic analysis provided new insights into possible structure-function relationships of Jmjd6 proteins and on the evolution of the whole superfamily of $\mathrm{DSBH} / \mathrm{JmjC}$ domain containing proteins. Of note, the observations made on the protein model are not experimentally proven at the current state and await confirmation when the crystallographic structure of Jmjd6 has been obtained. In addition, we provide first evidence for a counteracting transcriptional regulative mechanism involving the co-localised Jmjd6 and 1110005A03Rik genes.

\section{Methods}

\section{Bioinformatic approaches}

To investigate the splice variants of JmjdG, the genomic sequence of Jmjd6 and adjacent genes on either side was extracted to perform an unrestricted e2g-based annotation of mouse ESTs with a seed-length of 18 and a minimum identity of 95\% [67]. The resulting list of ESTs that overlap with the known Jmjd6 coding-region was passed on to a self-developed script that automatically retrieves the 
sequences of all matches and performs an in-depth verification of each EST using Sim4 [68]. The script sorts matches in different groups, according to the match quality. All matches with unidirectional splice sites, without internal gaps in the mRNA, and a $<95 \%$ identity score to the corresponding genomic regions leaving aside the polyA tail were used for manual annotation.

PipMaker sequence analysis [66] was done by extracting genomic sequences of 50 to $100 \mathrm{~kb}$ encompassing the Jmjd6 locus and neighbouring loci of mouse, human, bovine, armadillo, opossum, zebrafish, chicken, western clawed frog and puffer fish. Sequences were analysed by RepeatMasker [97] and positions of all coding sequences/ exons in JmjdG, 1110005A03Rik, and Sfrs2 were identified and annotated using the BLAST algorithm. The masked and annotated mouse sequence were then finally used for interspecies pairwise alignment with the sequences from the different species mentioned above using the program PipMaker [66].

To identify Jmjd6 orthologous sequences, a standard NCBI blastp-search against the $\mathrm{nr}$ database using amino acids 52-152 of the murine Jmjd6 protein was performed [98]. Identified sequences were validated using the "reciprocal best BLAST hit" criterion in a Mus musculus-restricted blastp-search against the nr database [72].

SMART-parser, a Perl-script for batch queries against SMART [99], data retrieval and processing was developed to identify proteins with similar domains as Jmjd6. SMART and SMART-parser were used to automatically identify, extract and annotate the sequences of all JmjC-, Pfam-JmjC, Blast-JmjC-, P4Hc-, Pfam-2OG-FeII-Oxy- and Pfam-PhyH-domains for later processing. These domain names have been used in the different public databases to indicate the presence of the $\mathrm{JmjC}$ domain containing DSBH fold proteins.

Multiple sequence alignments were generated employing ClustalW with a default setup and five iterations of realignment. Visualisation, calculation of a background frequency corrected sequence logo, and calculation of amino acid conservation was done using LaTeX and TEXshade [100]. A ClustalW alignment of all Jmjd6 orthologues (amino acids 44-329) identified in the prior approaches was used to generate a 3D-structure of Jmjd6 using the pipeline from the MPI Toolkit [101]. To add 2OG and $\mathrm{Fe}(\mathrm{II})$ to the predicted structure and the predicted active site of Jmjd6, an iterative backbone annealing of the Hif1an structure (1h2k) on top of the Jmjd6 model was performed using the SwissPDB Viewer [102]. Afterwards, the coordinates of $2 \mathrm{OG}$ and $\mathrm{Fe}$ (II) were added to the Jmjd6 model and energies were minimised using Deep-
View. 3D representations of molecules were rendered using PyMOL [103].

For a maximum likelihood phylogenetic analysis of Jmjd6 orthologues, we employed the PHYLIP toolbox [104]. A ClustalW alignment of all proteins with significant homology to Jmjd6 was used as an input for ProML, using the Jones-Taylor-Thornton algorithm with an estimated constant rate of mutations, global rearrangements and the more accurate iterated branch lengths option. The primary protein sequences of Jmjd6/JMJD6 were analysed for sumoylation sites using a SUMOplot prediction with a cutt-off of $90 \%$ probability [105]. Nuclear export signals (NES) were identified using the NetNES 1.1 server using a cut-off of 95\% identity to the NES Hidden Markow Model in the database [106]. Secondary structure predictions were generated using the Jpred web service [107].

\section{Identification of Jmjd6 splice variants}

RNA was isolated from different cells (HEK 293-T, A549, mouse embryonic fibroblasts (MEFs) and reverse transcribed. Murine splice variants were amplified using the forward primer 5'-GGTGGTGCCTCTTCCCAACAAA-3' and the reverse primers 5'-TCCAGGTCAGGGTTGGGACAC-3' and 5'-AGCTAGAAGAGTCGCTGGAGCTGTC-3'. The amplicons of the primer pairs had a product size of 363 bp (Mm3), 407 bp (Mm2), and $432 \mathrm{bp}$ $(\mathrm{Mm} 1)$, respectively. All fragments were analysed by sequencing and the sequences were submitted to GenBank (Acc. No. EF527404 and EF527405). Human cDNAs (C1244142, C1244152, C1244171-10, C1244035-10, C1244149, C1244264-10, C1244183, C1244246-10, C1244122-10, C1244260-10) were obtained from the BioChain Institute (Hayward, USA) and amplified according to the manufactures instructions using the forward primer 5'-GGGAACTCATCAAAGTGACCCGAGACG-3' and the reverse primers 5'-ACTGCCGCTGCCCGTGCTGTATC-3' and 5'-CGAGTCTGCGAGGACTGCCAACTCG-3'. The amplicons had a product size of $320 \mathrm{bp}$ (Hs2), $344 \mathrm{bp}$ (Hs1) and 438 bp (Hs3), respectively. All fragments were analysed by sequencing and the sequences were submitted to GenBank (Acc. No. EF527406 and EF527407).

\section{Expression analysis of Jmjd6, I I I 0005A03Rik and Sfrs2}

RNA was isolated and reverse transcribed. Expression of Jmjd6, 1110005A03Rik, Sfrs2 in embryos or cells were analysed using the primers 5'-GTT CCA GCT CGT CAG ACT CG-3' and 5'-TGC CCC TAA GAC ATG ACC AC-3' for Jmjd6, 5'-GCC ACC ACA AGA CAT CAT TCT TG-3' and 5'TCA GAT AAT TCA GCT TTA TGC CAG G-3' for 1110005A03Rik, 5'-GCT CCA GAT CAA CCT CCA AG-3' and 5'-GCC ACC TGA GGC AGA TTA AA-3' for Sfrs2, respectively. 


\section{Analysis of the bidirectional transcriptional unit} An 841 bp and an 840 bp fragment was amplified from mouse C57BL/6J genomic DNA using the forward 5'-CTCGAGCTCTCGTAGTAGTTGTGCCGGGT-3' and reverse 5'GCTAGCACTGTCCCTAAATGTGTCACTGGAGC-3' primer as well as the forward 3'-GCTAGCTCTCGTAGTAGTTGTGCCGGGT-5' and reverse 5'-CTCGAGACTGTCCCTAAATGTGTCACTGGAGC-3' primer, respectively. These fragments were integrated into a promoter-less luciferase expression vector (pGL3, Promega, Madison, USA) and these constructs were introduced into HEK 293-T cells, A549 cells or mouse embryonic fibroblasts (MEFs) using Nanofection (PAA, Pasching, Austria). After 24h of cultivation at $37^{\circ} \mathrm{C}$ and $5 \% \mathrm{CO}_{2}$, cell extracts were generated to measure the luciferase-activity using the DualLuciferase-Reporter-Assay System (Promega) according to the manufactures instructions. The obtained results were normalized to pGL4.74 (Promega) luciferase-activity. The measurements were performed in triplicate and repeated at least three times.

\section{Authors' contributions}

$\mathrm{PH}$ and JB designed the computational pipeline flow together. The domain based multiple sequence alignment analysis of all protein sequences, the phylogeny prediction there from and the PipMaker sequence analysis as well as the analysis of the different mouse mutant Jmjd6 alleles was carried out by JB. PH contributed the fulllength Jmjd6 analysis, 3D structure prediction and developed the new method for conservation based residue shading. Furthermore, $\mathrm{PH}$ identified and validated new splice variants of JmjdG, characterised the bi-directional Jmjd6 transcriptional unit and developed SMART-parser as well as additional Perl scripts for automated data processing. SE carried out parts of the Jmjd6 expression analysis. AL coordinated the study and wrote the paper. All authors read and approved the final manuscript.

\section{Additional material}

\section{Additional file 1}

Validation of JMJD6 splices variants in humans. RT-PCR expression analysis of JMJD6 splice variants in a human cDNA tissue panel obtained from the BioChain Institute (Hayward, USA). Arrows indicate amplicons from correctly predicted splice variants that were confirmed by sequencing. Multiplex PCR amplification results in one verified unspecific PCR fragment migrating at approximately $400 \mathrm{bp}$. Amplification of the housekeeping gene Ribosomal Protein 9 (RPS9) was used as loading control. Click here for file

[http://www.biomedcentral.com/content/supplementary/14712164-9-293-S1.pdf]

\section{Additional file 2}

Absence of nonsense-mediated decay in Jmjd6 splice variants. Two additional putative exons (green boxes) were identified (A). Alternative splicing using these two exons results in the generation of two additional transcripts of Jmjd6 (variants 2 and 3, respectively). Half arrows and orange lines in the schematic presentation of the transcripts highlight the combination of exons detected by RT-PCR in (B) and (C). Primers were designed to bind to the respective exons shown. (B) Experimental validation of the two new predicted alternative Jmjd6 transcripts using RT-PCR and agarose gel electrophoresis. Expression analyses confirmed that both splice variants are not subject to nonsense-mediated decay in adult mouse organs and (C) human tissues. Amplification of the housekeeping $\beta$-actin gene was used as a RNA loading control.

Click here for file

[http://www.biomedcentral.com/content/supplementary/14712164-9-293-S2.pdf]

\section{Additional file 3}

Comparison of strategies used to inactivate the Jmjd6 gene in mouse EScells. Schematic representation of the mouse locus according to the Ensembl annotation of the Jmjd6 gene and its neighbouring genes (A). Arrows indicate transcriptional orientation of the genes along chromosome 11 , from centromer to telomer. Jmjd6 exons are shown in green, exons of 1110005A03Rik in blue, and of Sfrs 2 in yellow. Individual exons are numbered. The strategies used to generate the different targeted knock out alleles are shown. Red boxes indicate gene regions deleted by homologous recombination. The Jmjd $6^{\mathrm{tm} 1 \mathrm{Fl} v}$ allele was generated by replacing an AatII/SpeI fragment by a neomycin resistance cassette. In the Jmjd $6^{\mathrm{tm} 1 \text { Ysfk }}$ allele the EcoRI fragment and in the Jmjd6 $6^{\mathrm{tm} 1 \mathrm{Gbf}}$ allele the AatII/RsrII fragment were replaced respectively. Based on these chosen fragments, targeted inactivation of Jmjd6 seems to affect in two of the three alleles the neighbouring 1110005A03Rik locus. (B) RT-PCR analysis of the expression of the Jmjd6, 1110005A03Rik (Rik) and Sfrs2 genes in Jmjd6 wild type and Jmjd6 homozygous mutant embryos. The expression of 1110005A03Rik is not detectable in the Jmjd6 ${ }^{\mathrm{tm} 1 \mathrm{Flv}}$ allele and in the Jmjd $6^{\mathrm{tm}} 1$ Ysfk allele, whereas expression of Sfrs2 2 is unaltered in all three mouse lines investigated (C) Semi-quantitative RT-PCR analysis of 1110005A03Rik expression in $\mathrm{Jmjd} 6^{\mathrm{tm}} 1 \mathrm{Gbf}$ wild type and homozygous mutant embryos (embryonic stage E13.5) and in fetal-liver derived macrophages shows that the level of expression is not altered due to the targeted inactivation of the Jmjd6 gene in this mouse line. RT-PCR expression analysis was performed in differential dilution steps of $c D N A$ material $(1,1: 10,1: 100)$. In all RT-PCR expression experiments amplification of the housekeeping gene $\beta$-actin severed as loading control. Click here for file

[http://www.biomedcentral.com/content/supplementary/14712164-9-293-S3.pdf] 


\section{Additional file 4}

Phylogenetic tree of live for identified putative Jmjd6 orthologs and their domain composition. Scientific classification of species with identified putative Jmjd6 orthologs was performed according to the NCBI taxonomy browser [108]. Schematic presentation of the grouping and categorisation of 62 species with identified Jmjd6 proteins. The individual species are shown on the left side, underlying colours highlight the kingdoms - animalia (red), plantae (green), protista (blue), fungi (yellow), and eubacteria (magenta), boxes on the right side represent the domain. The intermediate boxes show informative higher order ranks (e.g. genus, family, order, class, or phyla). On the left side, the domain composition for each protein is given. Length of polypeptides in amino acids (aa) are indicated on the top. Asterisks indicate partial or truncated protein sequences. Click here for file

[http://www.biomedcentral.com/content/supplementary/14712164-9-293-S4.pdf]

\section{Additional file 5}

Evolutionary conservation of surface exposed residues in the Jmjd6 protein model (stereoview). The surface model was computed using PyMol and coloured according to the sequence conservation code below in percent sequence identity in 54 analysed species. The coordinating Fe(II) of the catalytic triad is depicted in black, the co-substrate 2OG is shown as stick representation.

Click here for file

[http://www.biomedcentral.com/content/supplementary/14712164-9-293-S5.pdf]

\section{Additional file 6}

Phylogenetic relationship of Jmjd6 to the superfamily of JmjC-domain containing proteins. In total 313 sequences of DSBH/JmjC domain containing proteins from humans and model organisms (mouse, zebrafish, pufferfish, nematode, fruit fly, and yeast) were analysed for their relationship to different subgroups of JmjC domain containing proteins. JmjC domain sequences of the proteins were analysed by multiple sequence alignment and subsequent bootstrap analysis using ClustalW (1000 bootstrap trials, 111 seeds) and PHYLIP (ProML). The resulting unrouted tree based on sequence similarity within the JmjC domain revealed 25 evolutionary conserved protein subgroups. These are highlighted in different colours according to the colour code on the left side. Five proteins that could not be grouped into subgroups are indicated in red letters. H.s. = Homo sapiens, M.m. = Mus musculus, D.r. = Danio rerio, T.n. = Tetraodon nigroviridis, T.r. $=$ Takifugu rubripes, $D . m .=$ Drosophila melanogaster, C.e. $=$ Caenorhabditis elegans, $S . c .=$ Saccharomyces cerevisiae, S.p. $=$ Schizosaccharomyces pombe

Click here for file

[http://www.biomedcentral.com/content/supplementary/14712164-9-293-S6.pdf]

\section{Additional file 7}

Features of structurally resolved 2OG-Fe(II)-dependent dioxygenases in comparison to Jmjd6. Table describing features of structurally resolved and experimentally characterised 2OG-Fe(II)-dependent dioxygenases in comparison to Jmjd6.

Click here for file

[http://www.biomedcentral.com/content/supplementary/14712164-9-293-S7.pdf]

\section{Additional file 8}

Catalogue of JmjC/DSBH domain containing proteins. Table of 313 JmjC/DSBH domain containing protein used for the phylogenetic analysis.

Click here for file

[http://www.biomedcentral.com/content/supplementary/1471-

2164-9-293-S8.xls]

\section{Additional file 9}

Jmjd6 homologous proteins. Catalogue of 62 identified Jmjd6 homologous proteins.

Click here for file

[http://www.biomedcentral.com/content/supplementary/14712164-9-293-S9.xls]

\section{Acknowledgements}

We would like to thank Ivonne Wegener for excellent technical assistance. Dr. Richard Flavell (Yale University, New Haven, USA) and Dr. Yoshinori Fukui (Kyushu University, Fukuoka, Japan) for making available Jmjd6 tm IFlv knockout mice and RNA from Jmjd6tm I Ysfk mutant embryos, respectively. We are thankful to Dr. Bastian Pasche ( $\mathrm{HZI}$, Braunschweig) for giving critical comments on the manuscript. This work was supported by a grant from Deutsche Forschungsgemeinschaft (DFG LE 2130/2-I) to AL.

\section{References}

I. Takeuchi T, Yamazaki Y, Katoh-Fukui Y, Tsuchiya R, Kondo S, Motoyama J, Higashinakagawa T: Gene trap capture of a novel mouse gene, jumonji, required for neural tube formation. Genes Dev 1995, 9:1211-1222.

2. Balciunas D, Ronne $\mathrm{H}$ : Evidence of domain swapping within the jumonji family of transcription factors. Trends Biochem Sci 2000 , 25:274-276.

3. Clissold PM, Ponting CP: JmjC: cupin metalloenzyme-like domains in jumonji, hairless and phospholipase A2beta. Trends Biochem Sci 200I, 26:7-9.

4. Klose RJ, Kallin EM, Zhang Y: JmjC-domain-containing proteins and histone demethylation. Nat Rev Genet 2006, 7:7 15-727.

5. Takeuchi T, Watanabe Y, Takano-Shimizu T, Kondo S: Roles of jumonji and jumonji family genes in chromatin regulation and development. Dev Dyn 2006, 235:2449-2459.

6. Clifton IJ, McDonough MA, Ehrismann D, Kershaw NJ, Granatino N Schofield CJ: Structural studies on 2-oxoglutarate oxygenases and related double-stranded beta-helix fold proteins. J Inorg Biochem 2006, 100:644-669.

7. Dunwell JM, Culham A, Carter CE, Sosa-Aguirre CR, Goodenough PW: Evolution of functional diversity in the cupin superfamily. Trends Biochem Sci 200I, 26:740-746.

8. Schofield CJ, Zhang Z: Structural and mechanistic studies on 2oxoglutarate-dependent oxygenases and related enzymes. Curr Opin Struct Biol 1999, 9:722-731.

9. Trewick SC, McLaughlin PJ, Allshire RC: Methylation: lost in hydroxylation? EMBO Rep 2005, 6:3 I5-320.

10. Lee Y, Song AJ, Baker R, Micales B, Conway SJ, Lyons GE: Jumonji, a nuclear protein that is necessary for normal heart development. Circ Res 2000, 86:932-938.

II. Takeuchi T, Kojima M, Nakajima K, Kondo S: jumonji gene is essential for the neurulation and cardiac development of mouse embryos with a C3H/He background. Mech Dev 1999, 86:29-38.

12. Toyoda $M$, Kojima $M$, Takeuchi $T$ : Jumonji is a nuclear protein that participates in the negative regulation of cell growth. Biochem Biophys Res Commun 2000, 274:332-336.

13. Benevolenskaya EV, Murray HL, Branton P, Young RA, Kaelin WG Jr: Binding of pRB to the PHD protein RBP2 promotes cellular differentiation. Mol Cell 2005, 1 8:623-635.

14. Hsieh JC, Sisk JM, Jurutka PW, Haussler CA, Slater SA, Haussler MR, Thompson CC: Physical and functional interaction between 
the vitamin D receptor and hairless corepressor, two proteins required for hair cycling. J Biol Chem 2003, 278:38665-38674

15. Moraitis AN, Giguere V, Thompson CC: Novel mechanism of nuclear receptor corepressor interaction dictated by activation function 2 helix determinants. Mol Cell Biol 2002, 22:683|-684|.

16. Potter GB, Beaudoin GM 3rd, DeRenzo CL, Zarach JM, Chen SH, Thompson CC: The hairless gene mutated in congenital hair loss disorders encodes a novel nuclear receptor corepressor. Genes Dev 200 I, I 5:2687-270I

17. Ahmad W, Faiyaz ul Haque M, Brancolini V, Tsou HC, ul Haque S, Lam $H$, Aita VM, Owen J, deBlaquiere M, Frank J, et al:: Alopecia universalis associated with a mutation in the human hairless gene. Science 1998, 279:720-724.

18. Cichon S, Anker M, Vogt IR, Rohleder H, Putzstuck M, Hillmer A, Farooq SA, Al-Dhafri KS, Ahmad M, Haque S, et al.: Cloning, genomic organization, alternative transcripts and mutational analysis of the gene responsible for autosomal recessive universal congenital alopecia. Hum Mol Genet 1998, 7:167|- 1679

19. Zarach JM, Beaudoin GM 3rd, Coulombe PA, Thompson CC: The co-repressor hairless has a role in epithelial cell differentiation in the skin. Development 2004, I $31: 4189-4200$.

20. Martin $C$, Zhang $Y$ : The diverse functions of histone lysine methylation. Nat Rev Mol Cell Biol 2005, 6:838-849.

2I. Tsukada Y, Fang J, Erdjument-Bromage $\mathrm{H}$, Warren ME, Borchers $\mathrm{CH}$, Tempst $\mathrm{P}$, Zhang Y: Histone demethylation by a family of JmjC domain-containing proteins. Nature 2006, 439:8II-816

22. Chen Z, Zang J, Whetstine J, Hong X, Davrazou F, Kutateladze TG, Simpson M, Mao O, Pan CH, Dai S, et al:: Structural insights into histone demethylation by JMJD2 family members. Cell 2006 , I 25:69|-702.

23. Whetstine JR, Nottke A, Lan F, Huarte M, Smolikov S, Chen Z Spooner E, Li E, Zhang G, Colaiacovo M, Shi Y: Reversal of histone lysine trimethylation by the JMJD2 family of histone demethylases. Cell 2006, I 25:467-48I.

24. Klose RJ, Gardner KE, Liang G, Erdjument-Bromage H, Tempst P, Zhang Y: Demethylation of histone $\mathrm{H} 3 \mathrm{~K} 36$ and $\mathrm{H} 3 \mathrm{~K} 9$ by Rph I: a vestige of an H3K9 methylation system in Saccharomyces cerevisiae? Mol Cell Biol 2007, 27:395I-396I.

25. Tu S, Bulloch EM, Yang L, Ren C, Huang WC, Hsu PH, Chen CH, Liao $\mathrm{CL}, \mathrm{Yu} \mathrm{HM}$, Lo WS, et al:: Identification of histone demethylase in Sacchromyces cerevisiae. J Biol Chem 2007, 282: | 4262- | 427 |

26. Yamane K, Tateishi K, Klose RJ, Fang J, Fabrizio LA, Erdjument-Bromage $H$, Taylor-Papadimitriou J, Tempst $P$, Zhang $Y$ : PLU-I is an H3K4 demethylase involved in transcriptional repression and breast cancer cell proliferation. Mol Cell 2007, 25:80 I-8I 2 .

27. Cloos PA, Christensen J, Agger K, Maiolica A, Rappsilber J, Antal T, Hansen $\mathrm{KH}$, Helin $\mathrm{K}$ : The putative oncogene GASCI demethylates tri- and dimethylated lysine 9 on histone H3. Nature 2006, 442:307-3II.

28. Fodor BD, Kubicek S, Yonezawa M, O'Sullivan RJ, Sengupta R, PerezBurgos L, Opravil S, Mechtler K, Schotta G, Jenuwein T: Jmjd2b antagonizes $\mathrm{H} 3 \mathrm{~K} 9$ trimethylation at pericentric heterochromatin in mammalian cells. Genes Dev 2006, 20:।557-|562.

29. Klose RJ, Yamane K, Bae Y, Zhang D, Erdjument-Bromage H, Tempst $P$, Wong J, Zhang $Y$ : The transcriptional repressor JHDM3A demethylates trimethyl histone $\mathrm{H3}$ lysine 9 and lysine 36. Nature 2006, 442:312-316.

30. Wissmann M, Yin N, Muller JM, Greschik H, Fodor BD, Jenuwein T, Vogler C, Schneider R, Gunther T, Buettner R, et al:: Cooperative demethylation by JMJD2C and LSDI promotes androgen receptor-dependent gene expression. Nat Cell Biol 2007, 9:347-353.

31. Christensen J, Agger K, Cloos PA, Pasini D, Rose S, Sennels L, Rappsilber J, Hansen KH, Salcini AE, Helin K: RBP2 belongs to a family of demethylases, specific for tri-and dimethylated lysine 4 on histone 3. Cell 2007, I 28:1063-1076.

32. Klose RJ, Yan Q, Tothova Z, Yamane K, Erdjument-Bromage $H$ Tempst P, Gilliland DG, Zhang Y, Kaelin WG Jr: The retinoblastoma binding protein RBP2 is an H3K4 demethylase. Cell 2007, I 28:889-900.

33. Iwase $S$, Lan F, Bayliss $P$, de la Torre-Ubieta L, Huarte M, Qi HH, Whetstine JR, Bonni A, Roberts TM, Shi Y: The X-linked menta retardation gene SMCX/JARIDIC defines a family of histone H3 lysine 4 demethylases. Cell 2007, 128: 1077-1088.

34. Lee MG, Norman J, Shilatifard A, Shiekhattar R: Physical and functional association of a trimethyl H3K4 demethylase and Ring6a/MBLR, a polycomb-like protein. Cell 2007, I 28:877-887.

35. Liang G, Klose RJ, Gardner KE, Zhang Y: Yeast Jhd2p is a histone H3 Lys4 trimethyl demethylase. Nat Struct Mol Biol 2007, 14:243-245.

36. Seward DJ, Cubberley G, Kim S, Schonewald M, Zhang L, Tripet B, Bentley DL: Demethylation of trimethylated histone H3 Lys4 in vivo by JARIDI JmjC proteins. Nat Struct Mol Biol 2007, 14:240-242.

37. Eissenberg JC, Lee MG, Schneider J, Ilvarsonn A, Shiekhattar R, Shilatifard $A$ : The trithorax-group gene in Drosophila little imaginal discs encodes a trimethylated histone $\mathrm{H} 3$ Lys4 demethylase. Nat Struct Mol Biol 2007, 14:344-346.

38. Lee N, Zhang J, Klose RJ, Erdjument-Bromage $H$, Tempst $P$, Jones RS, Zhang $Y$ : The trithorax-group protein Lid is a histone $\mathbf{H} 3$ trimethyl-Lys4 demethylase. Nat Struct Mol Biol 2007, I 4:34I-343.

39. Secombe J, Li L, Carlos L, Eisenman RN: The Trithorax group protein Lid is a trimethyl histone $\mathrm{H} 3 \mathrm{~K} 4$ demethylase required for dMyc-induced cell growth. Genes Dev 2007, 21:537-55I.

40. Huarte M, Lan F, Kim T, Vaughn MW, Zaratiegui M, Martienssen RA, Buratowski S, Shi Y: The fission yeast JMJ2 reverses histone $H_{3}$ lysine 4 tri-methylation. J Biol Chem 2007, 282:2I662-2I670.

4I. Agger K, Cloos PA, Christensen J, Pasini D, Rose S, Rappsilber J, Issaeva I, Canaani E, Salcini AE, Helin K: UTX and JMJD3 are histone H3K27 demethylases involved in HOX gene regulation and development. Nature 2007, 449:73I-734.

42. De Santa F, Totaro MG, Prosperini E, Notarbartolo S, Testa G, Natol G: The histone $\mathrm{H} 3$ lysine-27 demethylase Jmjd3 links inflammation to inhibition of polycomb-mediated gene silencing. Cell 2007, I30: I083-1094.

43. Lan F, Bayliss PE, Rinn JL, Whetstine JR, Wang JK, Chen S, Iwase S, Alpatov R, Issaeva I, Canaani E, et al.: A histone H3 lysine 27 demethylase regulates animal posterior development. Nature 2007, 449:689-694.

44. Lee MG, Villa R, Trojer P, Norman J, Yan KP, Reinberg D, Di Croce L, Shiekhattar R: Demethylation of H3K27 regulates polycomb recruitment and H2A ubiquitination. Science 2007, 3 I 8:447-450

45. Yamane K, Toumazou C, Tsukada Y, Erdjument-Bromage H, Tempst P, Wong J, Zhang Y: JHDM2A, a JmjC-containing H3K9 demethylase, facilitates transcription activation by androgen receptor. Cell 2006, I 25:483-495.

46. Zofall M, Grewal SI: Swi6/HPI recruits a JmjC domain protein to facilitate transcription of heterochromatic repeats. Mol Cell 2006, 22:68I-692.

47. Huang Y, Fang J, Bedford MT, Zhang Y, Xu RM: Recognition of histone $\mathrm{H} 3$ lysine-4 methylation by the double tudor domain of JMJD2A. Science 2006, 31 2:748-75।.

48. Dann CE 3rd, Bruick RK, Deisenhofer J: Structure of factor-inhibiting hypoxia-inducible factor I: An asparaginyl hydroxylase involved in the hypoxic response pathway. Proc Natl Acad Sci USA 2002, 99:15351-15356.

49. Elkins JM, Hewitson KS, McNeill LA, Seibel JF, Schlemminger I, Pugh CW, Ratcliffe PJ, Schofield CJ: Structure of factor-inhibiting hypoxia-inducible factor (HIF) reveals mechanism of oxidative modification of HIF-I alpha. J Biol Chem 2003, 278: $1802-1806$

50. Schofield CJ, Ratcliffe PJ: Oxygen sensing by HIF hydroxylases. Nat Rev Mol Cell Biol 2004, 5:343-354.

5I. Falnes PO, Johansen RF, Seeberg E: AlkB-mediated oxidative demethylation reverses DNA damage in Escherichia coli. Nature 2002, 419:178-182.

52. Trewick SC, Henshaw TF, Hausinger RP, Lindahl T, Sedgwick B: Oxidative demethylation by Escherichia coli AlkB directly reverts DNA base damage. Nature 2002, 419:174-178.

53. Aravind L, Koonin EV: The DNA-repair protein AlkB, EGL-9, and leprecan define new families of 2-oxoglutarate- and irondependent dioxygenases. Genome Biol 200I, 2:RESEARCH0007.

54. Böse J, Gruber AD, Helming L, Schiebe S, Wegener I, Hafner M, Beales $M$, Kontgen $F$, Lengeling $A$ : The phosphatidylserine receptor has essential functions during embryogenesis but not in apoptotic cell removal. J Biol 2004, 3:15. 
55. Schneider JE, Böse J, Bamforth SD, Gruber AD, Broadbent C, Clarke $\mathrm{K}$, Neubauer S, Lengeling A, Bhattacharya S: Identification of cardiac malformations in mice lacking Ptdsr using a novel highthroughput magnetic resonance imaging technique. $B M C D e v$ Biol 2004, 4:16.

56. Fadok VA, Bratton DL, Rose DM, Pearson A, Ezekewitz RA, Henson PM: A receptor for phosphatidylserine-specific clearance of apoptotic cells. Nature 2000, 405:85-90.

57. Cikala M, Alexandrova O, David CN, Proschel M, Stiening B, Cramer $P, B o ̈ t t g e r A:$ The phosphatidylserine receptor from Hydra is a nuclear protein with potential $\mathrm{Fe}$ (II) dependent oxygenase activity. BMC Cell Biol 2004, 5:26.

58. Cui P, Qin B, Liu N, Pan G, Pei D: Nuclear localization of the phosphatidylserine receptor protein via multiple nuclear localization signals. Exp Cell Res 2004, 293: I54-163.

59. Krieser RJ, Moore FE, Dresnek D, Pellock BJ, Patel R, Huang A, Brachmann C, White K: The Drosophila homolog of the putative phosphatidylserine receptor functions to inhibit apoptosis. Development 2007, 134:2407-24|4.

60. Mitchell JE, Cretanovic M, Tibrewal N, Patel V, Colamonici OR, L MO, Flavell RA, Levine JS, Birge RB, Ucker DS: The presumptive phosphatidylserine receptor is dispensable for innate antiinflammatory recognition and clearance of apoptotic cells. Biol Chem 2006, 28 I:57 I8-5725.

61. Tibrewal N, Liu T, Li H, Birge RB: Characterization of the biochemical and biophysical properties of the phosphatidylserine receptor (PS-R) gene product. Mol Cell Biochem 2007, 304:119-125.

62. Li MO, Sarkisian MR, Mehal WZ, Rakic P, Flavell RA: Phosphatidylserine receptor is required for clearance of apoptotic cells. Science 2003, 302: I560-I563.

63. Kunisaki Y, Masuko S, Noda M, Inayoshi A, Sanui T, Harada M, Sasazuki T, Fukui Y: Defective fetal liver erythropoiesis and T lymphopoiesis in mice lacking the phosphatidylserine receptor. Blood 2004, 103:3362-3364.

64. Fackelmayer FO: Protein arginine methyltransferases: guardians of the Arg? Trends Biochem Sci 2005, 30:666-67I.

65. Bermingham JR Jr, Arden KC, Naumova AK, Sapienza C, Viars CS, Fu XD, Khotz J, Manley JL, Rosenfeld MG: Chromosomal localization of mouse and human genes encoding the splicing factors ASF/SF2 (SFRSI) and SC-35 (SFRS2). Genomics 1995, 29:70-79.

66. Schwartz S, Zhang Z, Frazer KA, Smit A, Riemer C, Bouck J, Gibbs R, Hardison R, Miller W: PipMaker - a web server for aligning two genomic DNA sequences. Genome Res 2000, 10:577-586.

67. Krüger J, Sczyrba A, Kurtz S, Giegerich R: e2g: an interactive webbased server for efficiently mapping large EST and CDNA sets to genomic sequences. Nucleic Acids Res 2004, 32:W30I-304.

68. Florea L, Hartzell G, Zhang Z, Rubin GM, Miller W: A computer program for aligning a cDNA sequence with a genomic DNA sequence. Genome Res 1998, 8:967-974.

69. Fadok VA, Xue D, Henson P: If phosphatidylserine is the death knell, a new phosphatidylserine-specific receptor is the bellringer. Cell Death Differ 200I, 8:582-587.

70. Huntley MA, Golding GB: Selection and slippage creating serine homopolymers. Mol Biol Evol 2006, 23:2017-2025

7I. Amrani N, Sachs MS, Jacobson A: Early nonsense: mRNA decay solves a translational problem. Nat Rev Mol Cell Biol 2006, 7:415-425

72. Tatusov RL, Koonin EV, Lipman DJ: A genomic perspective on protein families. Science 1997, 278:63|-637.

73. Hubbard TJ, Aken BL, Beal K, Ballester B, Caccamo M, Chen Y, Clarke L, Coates G, Cunningham F, Cutts T, et al.: Ensembl 2007. Nucleic Acids Res 2007, 35:D610-617.

74. Söding J: Protein homology detection by HMM-HMM comparison. Bioinformatics 2005, 21 1:95I-960.

75. Sali $A$, Blundell TL: Comparative protein modelling by satisfaction of spatial restraints. J Mol Biol I993, 234:779-8|5.

76. Britsch L, Dedio J, Saedler H, Forkmann G: Molecular characterization of flavanone 3 beta-hydroxylases. Consensus sequence, comparison with related enzymes and the role of conserved histidine residues. Eur J Biochem 1993, 2 17:745-754.

77. Hogan DA, Smith SR, Saari EA, McCracken J, Hausinger RP: Sitedirected mutagenesis of 2,4-dichlorophenoxyacetic acid/ alpha-ketoglutarate dioxygenase. Identification of residues involved in metallocenter formation and substrate binding. Biol Chem 2000, 275:12400-12409.
78. Lee DH, Jin SG, Cai S, Chen Y, Pfeifer GP, O'Connor TR: Repair of methylation damage in DNA and RNA by mammalian AlkB homologues. J Biol Chem 2005, 280:39448-39459.

79. Yu B, Edstrom WC, Benach J, Hamuro Y, Weber PC, Gibney BR, Hunt JF: Crystal structures of catalytic complexes of the oxidative DNA/RNA repair enzyme AlkB. Nature 2006, 439:879-884

80. Klose RJ, Zhang Y: Regulation of histone methylation by demethylimination and demethylation. Nat Rev Mol Cell Biol 2007, 8:307-3।8.

81. Ozer A, Bruick RK: Non-heme dioxygenases: cellular sensors and regulators jelly rolled into one? Nat Chem Biol 2007, 3:144-I53.

82. Weitzman JB: The curious world of apoptotic cell clearance. $J$ Biol 2004, 3: 13

83. Williamson P, Schlegel RA: Hide and seek: the secret identity of the phosphatidylserine receptor. J Biol 2004, 3: 14 .

84. Wolf A, Schmitz C, Bottger A: Changing story of the receptor for phosphatidylserine-dependent clearance of apoptotic cells. EMBO Rep 2007, 8:465-469.

85. Aravind L, Landsman D: AT-hook motifs identified in a wide variety of DNA-binding proteins. Nucleic Acids Res 1998 , 26:44I3-442I.

86. Chang $B$, Chen $Y$, Zhao $Y$, Bruick RK: JMJD6 is a histone arginine demethylase. Science 2007, 3 I 8:444-447.

87. Guccione E, Bassi C, Casadio F, Martinato F, Cesaroni M, Schuchlautz $\mathrm{H}$, Luscher B, Amati B: Methylation of histone H3R2 by PRMT6 and $\mathrm{H} 3 \mathrm{~K} 4$ by an MLL complex are mutually exclusive. Nature 2007, 449:933-937.

88. Kirmizis A, Santos-Rosa H, Penkett CJ, Singer MA, Vermeulen M, Mann M, Bahler J, Green RD, Kouzarides T: Arginine methylation at histone H3R2 controls deposition of H3K4 trimethylation. Nature 2007, 449:928-932.

89. Ng SS, Kavanagh KL, McDonough MA, Butler D, Pilka ES, Lienard BM, Bray JE, Savitsky P, Gileadi O, von Delft F, et al.: Crystal structures of histone demethylase JMJD2A reveal basis for substrate specificity. Nature 2007, 448:87-91.

90. Kim J, Daniel J, Espejo A, Lake A, Krishna M, Xia L, Zhang Y, Bedford MT: Tudor, MBT and chromo domains gauge the degree of lysine methylation. EMBO Rep 2006, 7:397-403.

91. Li H, llin S, Wang W, Duncan EM, Wysocka J, Allis CD, Patel DJ: Molecular basis for site-specific read-out of histone H3K4me3 by the BPTF PHD finger of NURF. Nature 2006, 442:91-95.

92. Pena PV, Davrazou F, Shi X, Walter KL, Verkhusha VV, Gozani O, Zhao R, Kutateladze TG: Molecular mechanism of histone H3K4me3 recognition by plant homeodomain of ING2. Nature 2006, 442: $100-103$

93. Shi X, Hong T, Walter KL, Ewalt M, Michishita E, Hung T, Carney D, Pena P, Lan F, Kaadige MR, et al: ING2 PHD domain links histone H3 lysine 4 methylation to active gene repression. Nature 2006, 442:96-99.

94. Wysocka J, Swigut T, Xiao H, Milne TA, Kwon SY, Landry J, Kauer M, Tackett A], Chait BT, Badenhorst P, et al:: A PHD finger of NURF couples histone $\mathrm{H} 3$ lysine 4 trimethylation with chromatin remodelling. Nature 2006, 442:86-90.

95. Hong JR, Lin GH, Lin CJ, Wang WP, Lee CC, Lin TL, Wu JL: Phosphatidylserine receptor is required for the engulfment of dead apoptotic cells and for normal embryonic development in zebrafish. Development 2004, I $31: 5417-5427$.

96. Wang X, Wu YC, Fadok VA, Lee MC, Gengyo-Ando K, Cheng LC Ledwich D, Hsu PK, Chen JY, Chou BK, et al.: Cell corpse engulfment mediated by $C$. elegans phosphatidylserine receptor through CED-5 and CED-12. Science 2003, 302:1563-1566.

97. RepeatMasker [http://www.repeatmasker.org]

98. NCBI-BLAST [http://www.ncbi.nlm.nih.gov/BLAST]

99. SMART [http://smart.embl-heidelberg.de]

100. Beitz E: TEXshade: shading and labeling of multiple sequence alignments using LATEX2 epsilon. Bioinformatics 2000, 16:135-139.

10I. Biegert A, Mayer C, Remmert M, Soding J, Lupas AN: The MPI Bioinformatics Toolkit for protein sequence analysis. Nucleic Acids Res 2006, 34:W335-339.

102. Swiss-Pdb-Viewer [http://expasy.org/spdbv]

103. PyMOL [http://pymol.sourceforge.net/]

104. PHYLIP [http://evolution.genetics.washington.edu/phylip.html] 
105. SUMOplot [http://www.abgent.com.cn/doc/sumoplot/login.asp]

106. NetNES [http://www.cbs.dtu.dk/services/NetNES]

107. Jpred-3 [http://www.compbio.dundee.ac.uk/ www-jpred]

108. NCBI-Taxonomy [http://www.ncbi.nlm.nih.gov/Taxonomy/]

Publish with Biomed Central and every scientist can read your work free of charge

"BioMed Central will be the most significant development for disseminating the results of biomedical research in our lifetime. " Sir Paul Nurse, Cancer Research UK

Your research papers will be:

- available free of charge to the entire biomedical community

- peer reviewed and published immediately upon acceptance

- cited in PubMed and archived on PubMed Central

- yours - you keep the copyright

Submit your manuscript here:

http://www.biomedcentral.com/info/publishing_adv.asp
BioMedcentral 